Article

\title{
Deep Convolutional Neural Networks Capabilities for Binary Classification of Polar Mesocyclones in Satellite Mosaics
}

\author{
Mikhail Krinitskiy ${ }^{1, *(0)}$, Polina Verezemskaya ${ }^{1,2}{ }^{\oplus}$, Kirill Grashchenkov ${ }^{1,3}$, Natalia Tilinina ${ }^{1}(\mathbb{}$, \\ Sergey Gulev ${ }^{1}$ and Matthew Lazzara ${ }^{4}$ \\ 1 Shirshov Institute of Oceanology, Russian Academy of Sciences, Moscow 117997, Russia; \\ verezem@sail.msk.ru (P.V.); kg060296@mail.ru (K.G.); tilinina@sail.msk.ru (N.T.); gul@sail.msk.ru (S.G.) \\ 2 Research Computing Center of Lomonosov Moscow State University, Moscow 119234, Russia \\ 3 Moscow Institute of Physics and Technology, Moscow 141701, Russia \\ 4 University of Wisconsin-Madison and Madison Area Technical College, \\ Madison, WI 53704, USA; mattl@ssec.wisc.edu \\ * Correspondence: krinitsky@sail.msk.ru; Tel.: +7-926-141-6200
}

Received: 15 August 2018; Accepted: 28 October 2018; Published: 31 October 2018

\begin{abstract}
Polar mesocyclones (MCs) are small marine atmospheric vortices. The class of intense MCs, called polar lows, are accompanied by extremely strong surface winds and heat fluxes and thus largely influencing deep ocean water formation in the polar regions. Accurate detection of polar mesocyclones in high-resolution satellite data, while challenging, is a time-consuming task, when performed manually. Existing algorithms for the automatic detection of polar mesocyclones are based on the conventional analysis of patterns of cloudiness and they involve different empirically defined thresholds of geophysical variables. As a result, various detection methods typically reveal very different results when applied to a single dataset. We develop a conceptually novel approach for the detection of MCs based on the use of deep convolutional neural networks (DCNNs). As a first step, we demonstrate that DCNN model is capable of performing binary classification of $500 \times 500 \mathrm{~km}$ patches of satellite images regarding MC patterns presence in it. The training dataset is based on the reference database of MCs manually tracked in the Southern Hemisphere from satellite mosaics. We use a subset of this database with MC diameters falling in the range of $200-400 \mathrm{~km}$. This dataset is further used for testing several different DCNN setups, specifically, DCNN built "from scratch", DCNN based on VGG16 pre-trained weights also engaging the Transfer Learning technique, and DCNN based on VGG16 with Fine Tuning technique. Each of these networks is further applied to both infrared (IR) and a combination of infrared and water vapor (IR + WV) satellite imagery. The best skills ( $97 \%$ in terms of the binary classification accuracy score) is achieved with the model that averages the estimates of the ensemble of different DCNNs. The algorithm can be further extended to the automatic identification and tracking numerical scheme and applied to other atmospheric phenomena that are characterized by a distinct signature in satellite imagery.
\end{abstract}

Keywords: deep learning; convolutional neural networks; polar mesocyclones; satellite data processing; pattern recognition

\section{Introduction}

Polar mesoscale cyclones (MCs) are high-latitude marine atmospheric vortices. Their sizes range from 200 to $1000 \mathrm{~km}$ with lifetimes typically spanning from 6 to $36 \mathrm{~h} \mathrm{[1].} \mathrm{A} \mathrm{specific} \mathrm{intense} \mathrm{type} \mathrm{of}$ mesocyclones, the so-called polar lows (PLs), is characterized by surface winds of more than $15 \mathrm{~m} / \mathrm{s}$ and strong surface fluxes. These PLs have a significant impact on local weather conditions causing 
rough seas. Being relatively small in size (compared to the extratropical cyclones), PLs contribute significantly to the generation of extreme air-sea fluxes and initialize intense surface transformation of water masses resulting in the formation of ocean deep water [2-4]. These processes are most intense in the Weddel and Bellingshausen Seas in the Southern Hemisphere (SH) and in the Labrador, Greenland, and Irminger Seas in the Northern Hemisphere (NH).

One potential source of data is reanalyses. However, MCs, being critically important for many oceanographic and meteorological applications, are only partially detectable in different reanalysis datasets, primarily due to the inadequate resolution. Studies $[3,5-8]$ have demonstrated the significant underestimation of both number of mesocyclones and wind speeds by modern reanalyses in contrast with satellite observations of MCs cloud signatures and wind speeds. This hints that the spatial resolution of modern reanalyses is still not good enough for reliable and accurate detection of MCs. Press et al. argued for at least $10 \times 10$ grid points is necessary for effective capturing the MC [9]. This implies a $30 \mathrm{~km}$ spatial resolution in the model or reanalysis is needed for detecting MC with the diameter of $300 \mathrm{~km}$. Some studies [5,10] have demonstrated that $80 \%(64 \%)$ of MCs (PLs) in the $\mathrm{SH}(\mathrm{NH})$ are characterized by the diameters ranging from 200 to $500 \mathrm{~km}$ ( 250 to $450 \mathrm{~km}$ for NH in [10]). The most recent study of Smirnova and Golubkin [11] revealed that only $70 \%$ of those could be sustainably represented, even in the very high-resolution Arctic System Reanalysis (ASR) [12]. At the same time only $53 \%$ of the observed MCs characterized by diameters less than $200 \mathrm{~km} \mathrm{[5]} \mathrm{are}$ sustainably represented in ASR [11]. It was also shown [3,5,6] that both number of MCs and associated winds in modern reanalyses are significantly underestimated when compared to satellite observations of cloud signatures of MCs and satellite scatterometer observations of MC winds.

One might argue for the use of operational analyses for detecting MCs. However, these products are influenced by the changes in the numerics of a model and physics parameterization schemes with newly developed ones, and by the changes of the performance of data assimilation system and the amount of assimilated data. This leads to artificial trends at climatological timescales. In several studies, automated cyclone tracking algorithms that were originally developed for mid-latitude cyclones were adapted for MCs identification and tracking [13-15]. These algorithms were applied to the preprocessed (spatially filtered) reanalysis data and delivered climatological assessments of MCs activity in reanalyses or revealed the direction for their improvement. However, reported estimates of MCs numbers, sizes, and lifecycle characteristics vary significantly in these studies.

Zappa et al. [13] shows that ECMWF operational analysis makes it possible to detect up to $70 \%$ of the observed PLs, which is higher than ERA40 and ERA-Interim reanalyses (24\%, $45 \%$, or $55 \%$ depending on the procedure of tracking and the choice of reanalysis $[6,13])$. One bandpass filter in conjunction with different combinations of criteria used for the post-processing of the MC tracking results may result in a 30\% spread in the number of PLs [13]. Observational satellite-based climatologies of MCs and PLs [5,10,16-19] consistently reveal a mean vortex diameter of 300-350 km. In a number of reanalysis-based automated studies [14,20], the upper limit of MC and PL diameters was set to $1000 \mathrm{~km}$, resulting in the mean values between 500 and $800 \mathrm{~km}$. Thus, the estimates of MC sizes are still inconsistently derived with automated tracking algorithms. This inconsistency contrasts with the estimates for midlatitude cyclones' characteristics derived with the ensemble of tracking schemes [21] applied to a single dataset.

Satellite imagery of cloudiness is another data source for identification and tracking of MCs. These data allow for visual identification of cloud signatures that are associated with MCs. However, the manual procedure re Carleton quires enormous effort to build a long enough dataset. Pioneering work of Wilhelmsen [22] used ten years of consecutive synoptic weather maps, coastal observational stations, and several satellite images over the Norwegian and Barents Seas to describe local PLs activity. Later, in the 1990s, the number of instruments and satellite crossovers increased. It provoked many studies [16,23-28] evaluating the characteristics of MCs occurrence and lifecycle in different regions of both $\mathrm{NH}$ and $\mathrm{SH}$. These studies identified major MCs generation regions, their dominant migration directions, and cloudiness signature types that are associated with MCs. Increases in the amount of 
satellite observations allowed for the development of robust regional climatologies of MCs occurrence and characteristics. For the SH, Carleton [27] used twice daily cloudiness imagery of West Antarctica and classified for the first time four types of cloud signatures associated with PLs (comma, spiral, transitional type, and merry-go-round). This classification has been confirmed later in many works and it is widely used now. Harold et al. $[16,26]$ used daily satellite imagery for building one of the most detailed datasets of MC characteristics for the Nordic Seas (Greenland, Norwegian, Iceland, and Northern Seas). Also, Harold et al. $[16,26]$ developed a detailed description of the conventional methodology for the identification and tracking of MCs using satellite IR imageries.

There are also several studies regarding polar MCs and PLs activity in the Sea of Japan. Gang et al. [29] conducted the first long-term (three winter months) research of PLs in the Sea of Japan based on visible and IR imagery from the geostationary satellite with hourly resolution. In the era of multi-sensor satellite observations, Gurvich and Pichugin [30] developed the nine-year climatology of polar MCs based on water vapor, cloud water content and surface wind satellite data over the Western Pacific. This study reveals a mean MCs diameter of $200-400 \mathrm{~km}$ as well.

As these examples illustrate, most studies of MCs activity are regional $[10,17,18,31,32]$ and they cover relatively short time periods [5] due to the very costly and time-consuming procedure of visual identification and tracking of MCs. Thus, development of the reliable long-term (multiyear) dataset covering the whole circumpolar Arctic or Antarctic remains a challenge.

Recently, machine learning methods have been found to be quite effective for the classification of different cloud characteristics such as solar disk state and cloud types. There are studies in which different machine learning techniques are used for recognizing cloud types [33-35]. Methodologies employed include deep convolutional neural networks (DCNNs [36,37]), k-nearest-neighbor classifier (KNN), and Support Vector Machine (SVM) and fully-connected neural networks (FCNNs). Krinitskiy [38] used FCNNs for the detection of solar disk state and reported very high accuracy $(96.4 \%)$ of the proposed method. Liu et al. [39] applied DCNNs to the fixed-size multichannel images to detect extreme weather events and reported the success score of the detection of 89 to $99 \%$. Huang et al. [40] applied the neural network "DeepEddy" to the synthetic aperture radar images for detection of ocean meso- and submesoscale eddies. Their results are also characterized by high accuracy exceeding $96 \%$ success rate. However, Deep Learning (DL) methods have never been applied for detecting MCs.

DCNNs are known to demonstrate high skills in classification, pattern recognition, and semantic segmentation, when applied to two-dimensional (2D) fields, such as images. The major advantage of DCNNs is the depth of processing of the input 2D field. Similarly to the processing levels of satellite data (L0, L1, L2, L3, etc.), which allow retrieving, e.g., wind speed (L2 processing) from the raw remote measurements (L0), DCNNs are dealing with multiple levels of subsequent non-linear processing of an input image. In contrast to the expert-designed algorithms, the neural network levels of processing (so-called layers) are built in a manner that is common within each specific layer type (convolutional, fully-connected, subsampling, etc.). During the network training process, these layers of a DCNN acquire the ability to extract a broad set of patterns of different scales from the initial data [41-44]. In this sense, a trained DCNN closely simulates the visual pattern recognition process naturally used by a human operator. There exist several state-of-the-art network architectures, such as "AlexNet" [35], "VGG16" and "VGG19" [45], "Inception" of several subversions [46], "Xception" [47], and residual networks [48]. Each of these networks has been trained and tested using a range of datasets, including the one that is considered as a "reference" for the further image processing, the so-called ImageNet [49]. Continuous development of all DCNNs aims to improve the accuracy of the ImageNet classification. Today, the existing architectures demonstrate high accuracy with the error rate from $2 \%$ to $16 \%$ [50].

A DCNN by design closely simulates the visual recognition process. IR and WV satellite mosaics can be interpreted as images. Thus, assuming that a human expert detects MCs on these mosaics on the basis of his visual perception, application of DCNN appears to be a promising approach to this problem. Liu et al. [39] described a DCNN applied to the detection of tropical cyclones and atmospheric rivers in the $2 \mathrm{D}$ fields of surface pressure, temperature and precipitation stacked together 
into "image patches". However, the proposed approach cannot be directly applied to the MC detection. This method is skillful for the detection of large-scale weather extremes that are discernible in reanalysis products. However, as noted above, MCs have a poorly observable footprint in geophysical variables of reanalyses.

In this study, we apply the DL technique [51-53] to the satellite IR and WV mosaics distributed by Antarctic Meteorological Research Center [54,55]. This allows for the automated recognition of MCs cloud signatures. Our focus here is exclusively on the capability of DCNNs to perform a binary classification task regarding MCs patterns presence in patches of satellite imagery of cloudiness and/or water vapor, rather than on the DCNN-based MC tracking. This will indicate that a DCNN is capable of learning the hidden representation that is in accordance with the data and the MCs detection problem.

The paper is organized as follows. Section 2 describes the source data based on MC trajectories database [5]. Section 3 describes the development of the MC detection method that is based on deep convolutional neural networks and necessary data preprocessing. In Section 4, we present the results of the application of the developed methodology. Section 5 summarizes the paper with the conclusions and provides an outlook.

\section{Data}

For the training of DCNNs, we use the MCs dataset for the Southern Ocean (SOMC, http:/ / sail.ocean.ru/antarctica/), consisting of $1735 \mathrm{MC}$ trajectories, resulting in $9252 \mathrm{MC}$ locations and associated estimates of MC sizes [5] for the four-months period (June, July, August, September) of 2004 (Figure 1a). The dataset was developed by visual identification and tracking of MCs using 976 consecutive three-hourly satellite IR (10.3-11.3 micron) and WV ( 6.7 microns) mosaics provided by the Antarctic Meteorological Research Center (AMRC) Antarctic Satellite Composite Imagery (AMRC ASCI) [54,55]. These mosaics are available online (https:/ / amrc.ssec.wisc.edu/data/) and are composites of geostationary and polar-orbiting satellite observations (GOES East and West, Meteosat, MTSAT, NOAA satellites, METOP, FY-2, Aqua, Terra etc.). This mosaics dataset is maintained by the AMRC [55]. The SOMC dataset contains longitudes and latitudes of MC centers at each three-hourly time step of the MC track, as well as MC diameter and the cloudiness signature type through the MC life cycle [5]. These characteristics were used along with the associated cloudiness patterns of MCs from the initial IR and WV mosaics for training DCNNs.
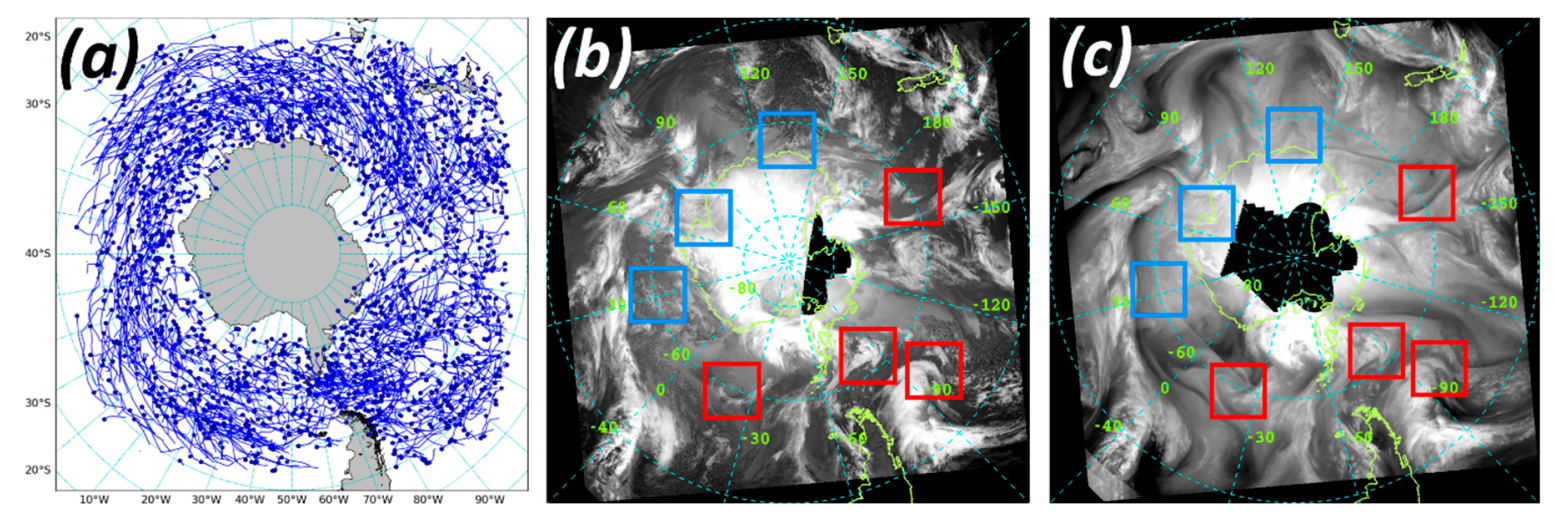

Figure 1. The input for the deep convolutional neural networks (DCNNs). (a) Trajectories of all mesocyclones (MCs) in Southern Ocean MesoCylones (SOMC) dataset, blue dots mark the point of generation of MC. Snapshots of satellite mosaics for SH for (b) InfraRed (IR) and (c) Water Vapor (WV) channels at 00:00 UTC 02/06/2004. The red/blue squares indicate patches centered over the MCs (red squares) and those having no MC cloudiness signature in (blue) being cut from the mosaics for DCNNs training.

AMRC ASCI mosaics spatially combine observations from geostationary and polar-orbiting satellites and cover the area to the South of $\sim 40^{\circ} \mathrm{S}$ with three-hourly temporal and $5 \mathrm{~km}$ spatial 
resolution (Figure 1b,c). While the IR channel is widely used for MCs identification [16,17,26,27,32], we also additionally employ the WV channel imagery that provides a better accuracy over the ice-covered ocean, where the IR images are potentially incorrect.

\section{Methodology}

\subsection{Data Preprocessing}

For training models, we first co-located a square (patch) of $100 \times 100$ mosaic pixels $(500 \times 500 \mathrm{~km})$ with each MC center location from SOMC dataset (9252 locations in total) (Figure 2a-d). Since the distance between MCs in the multiple systems, such as the merry-go-round pattern, may be comparable to each MC diameter, and to ensure that (i) each patch covers only one MC and (ii) covers it completely, we require that MC diameters fall into $200-400 \mathrm{~km}$ range. Hereafter, we call this set of samples 'the true samples'. The chosen set of true samples includes $67 \%$ of the whole population of samples in SOMC dataset.

We additionally built the set of 'false samples' for DCNNs training. False samples were generated from the patches that do not consist of MC-associated cloudiness signatures (Figure 2e-h) according to the SOMC dataset. Table 1 summarizes the numbers of true and false samples that both make up the source dataset for our further analysis of IR and WV mosaics. The total number of snapshots used (both IR and WV) is 11,189. The true samples are 6177 (55\%) of them, and $5012(45 \%)$ are the false samples (see Figure 2). In order to unify images in the dataset, we normalized them by the maximum and the minimum brightness temperature (in the case of IR) over the whole dataset:

$$
x_{\text {norm }}=\frac{x-\min (X)}{\max (X)-\min (X)},
$$

where $x$ denotes the individual sample (represented by a matrix of $100 \times 100$ pixels) and $X$ is the whole dataset of 11,189 IR snapshots. The same normalization was applied to WV snapshots.

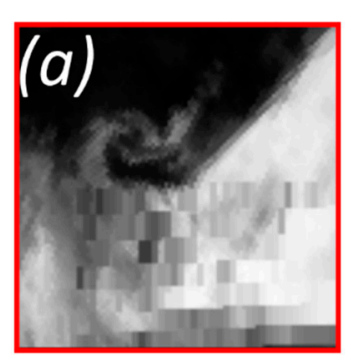

\section{TRUE samples (MC presented)}
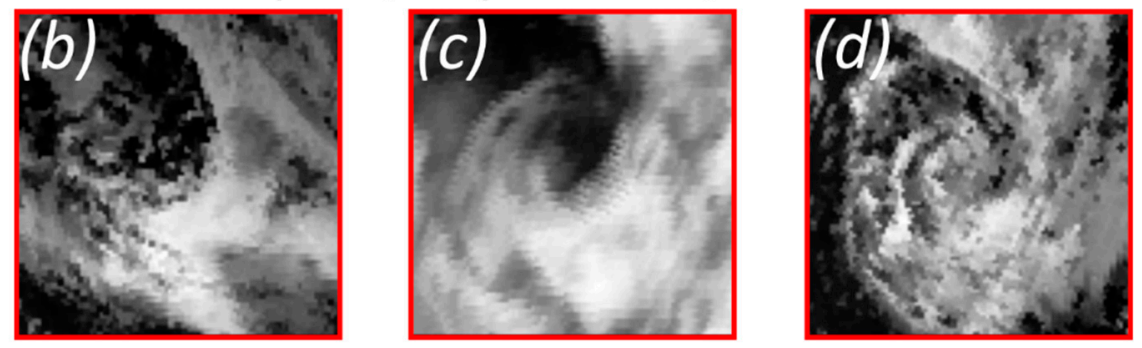

\section{FALSE samples (no MC presented)}
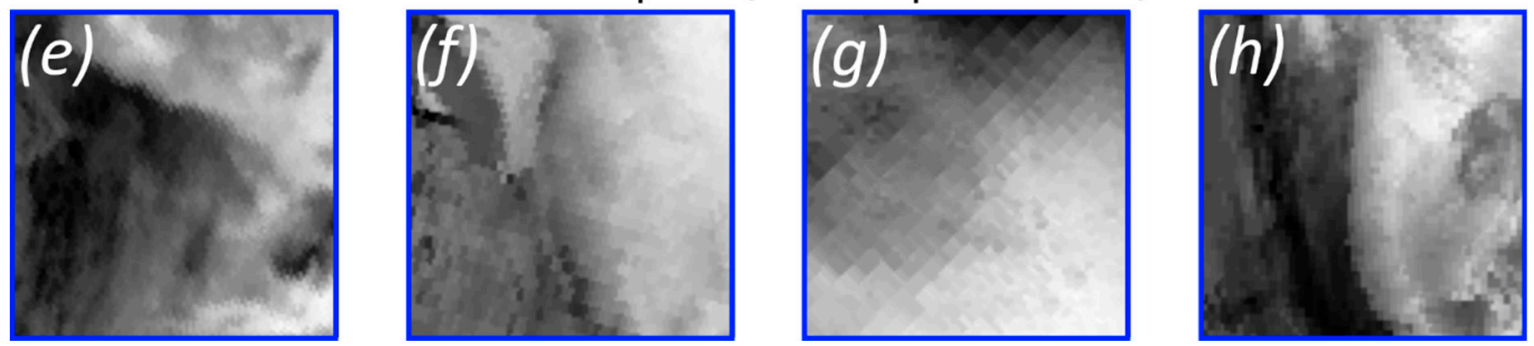

Figure 2. Examples (IR only) of true and false samples for DCNNs training and testing of DCNNs results assessment. $100 \times 100$ grid points $(500 \times 500 \mathrm{~km})$ patches of IR mosaics for $(\mathbf{a}-\mathbf{d})$ true samples and false (e-h) samples. 


\subsection{Formulation of the Problem}

We consider MC identification as a binary classification problem. We use the set of true and false samples (Figure 2) as input ("objects" herein). We have developed two DCNN architectures following two conditional requirements: either (i) the object is described by the IR image only or (ii) the object is described by both IR and WV images. Since the training dataset is almost target-balanced (see Table 1), assuming $\sim 50 / 50$ ratio of true/false samples, we further use the accuracy score as the measure of the classification quality. The accuracy score cannot be used as a reliable quality measure of any machine learning method in the case of the unbalanced training dataset. For example, in the case of a highly unbalanced dataset with the true/false ratio being $95 / 5$, it is easy to achieve $95 \%$ accuracy score by just forcing the model to produce only the true outcome. Thus, balancing the source dataset with false samples is critical for building the reliable classification model.

Table 1. Total number of true and false samples.

\begin{tabular}{cccc}
\hline & True Samples & False Samples & Total Samples \\
\hline IR & $6177(55 \%)$ & $5012(45 \%)$ & $11,189(100 \%)$ \\
WV & $6177(55 \%)$ & $5012(45 \%)$ & $11,189(100 \%)$ \\
\hline
\end{tabular}

\subsection{Justification of Using DCNN}

There is a set of best practices commonly used to construct DCNNs for solving classification problems [56]. While building and training DCNNs for MCs identifications, we applied the technique that was proposed by LeCun [41]. This technique implies the usage of consecutive convolutional layers that detect spatial data patterns, alternating with subsampling layers, which reduce the sample dimensions. The set of these layers is followed by a set of so-called fully-connected (FC) layers representing a neural classifier. The whole model built in this manner represents a non-linear classifier that is capable of directly predicting a target value for the input sample. A very detailed description of this model architecture can be found in [41]. We will further term the FC layers set as "FC classifier", and the preceding part containing convolutional and pooling layers as "convolutional core" (see Figures 3 and 4). The outcome of the whole model is the probability of MC presence in the input sample.

While handling multiple concurrent and spatially aligned geophysical fields, it is important to choose a suitable approach. LeCun [41] proposed the DCNN focused on the processing of only grayscale images-meaning just one 2D field. In order to handle multiple 2D fields, they may be stacked together to form a three-dimensional (3D) matrix by analogy with colorful images which have three color channels: red, green and blue. This approach can be applied when one uses pre-trained networks like AlexNet [36], VGG16 [45], ResNet [48] or similar architectures because of the original purpose of these networks to classify colorful images. However, this approach should be exploited carefully when applied to geophysical fields, because the mentioned networks were trained using massive datasets (e.g., ImageNet) of real photographed scenes, which means specific dependencies laying between channels (red, green and blue) within each image. In contrast to the stacking approach applied by Liu et al. [39], we use separate CNN branch for each channel (IR and WV) to ensure that we are not limiting the overall quality of the whole network (see Figure 4). In the following, we describe in detail each DCNN architecture for both cases: IR + WV (Figure 4) and IR alone (Figure 3).

Since we consider the binary classification, and the source dataset is almost target-balanced (see Table 1), we use as a quality measure the accuracy score or Acc, which is a rate of objects, classified correctly as compared to the ground truth: 


$$
A c c=\frac{1}{\|\mathcal{T}\|} \sum_{\mathcal{T}}\left[\hat{y}_{i}=y_{i}\right]
$$

where $\mathcal{T}$ denotes the dataset and $\|\mathcal{T}\|$ is its total samples count; $y_{i}$ is expert-defined target value (ground truth), $\hat{y}_{i}$ is the model decision whether the $i$-th object contain MC.

In addition to the baseline that is the network proposed in [41], we applied a set of additional approaches that are commonly used to improve the DCNN accuracy and generalization ability (see Appendix A). Specifically, we used Transfer Learning (TL) [57-62] with the VGG16 [45] network pre-trained on ImageNet [49] dataset; Fine Tuning (FT) [63], Dropout (Do) [64], and dataset augmentation (DA) [65] (see Appendix A). With these techniques applied in various combinations, we constructed six DCNN architectures that are summarized in Table 2. All of these architectures are built in a common manner: the FC classifier follows the one- (for IR only) or two-branched (for $\mathrm{IR}+\mathrm{WV}$ ) convolutional core. If the convolutional core is one-branched, its output itself is input data for the corresponding FC classifier. If the convolutional core is two-branched, the concatenation product of their outputs is the input data for the corresponding FC classifier. The very detailed description of the constructed architectures is presented in Appendix A. For each DCNN structure, we trained a set of models, as described in detail in Section 3.5. We also applied ensemble averaging (see Appendix A) of a set of models of identical configuration via averaging probabilities of true class for each object of the dataset. We term these six ensemble-averaged models the "second-order" models. We also applied ensemble averaging per sample of all trained DCNNs that were trained in this work. We term this model the "third-order" model. Each of these models was trained using the method of backpropagation of error (BCE loss, see Appendix A) [66], denoted as "backprop training" in Figures 3 and 4.

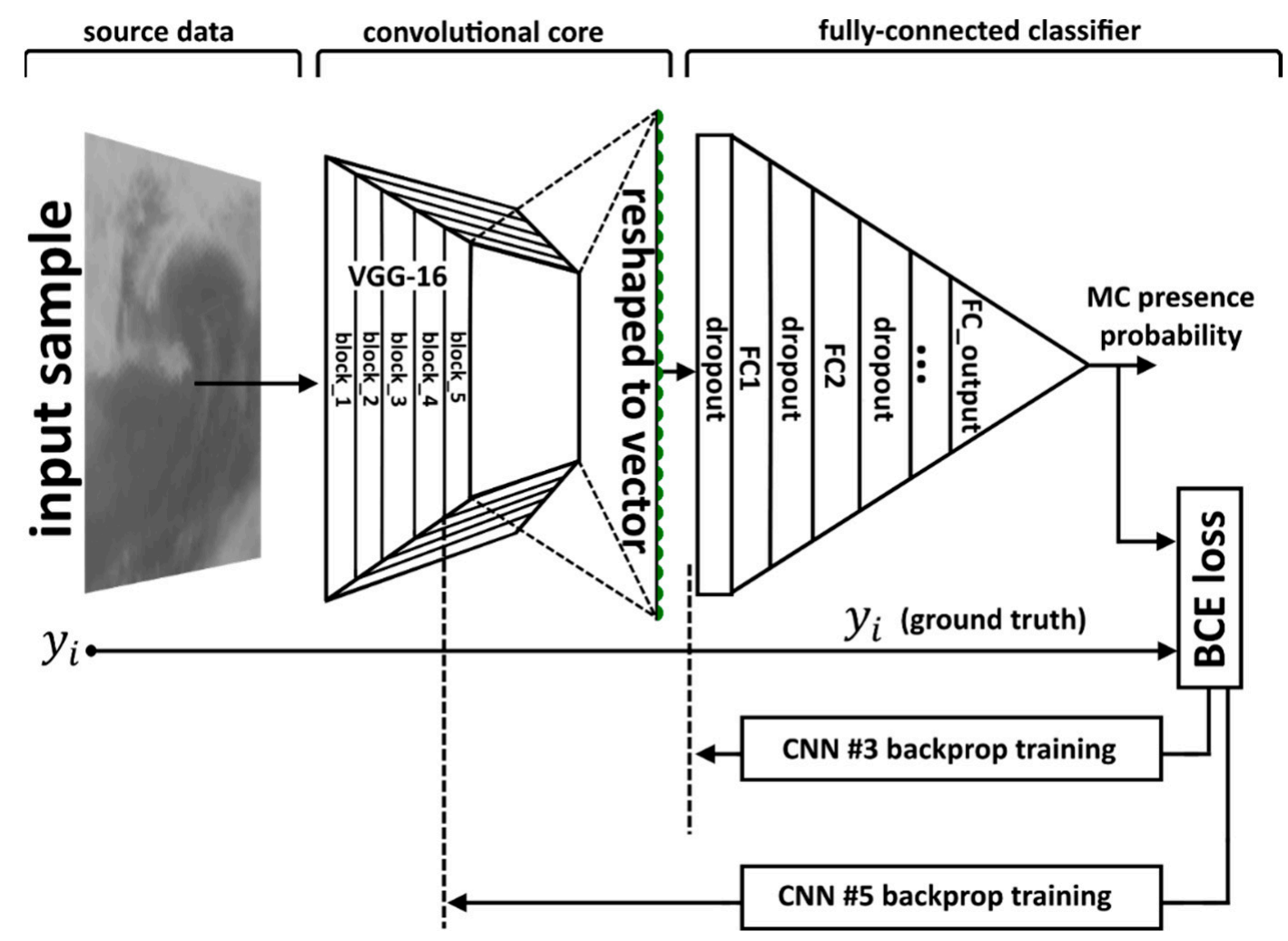

Figure 3. Convolutional neural networks $(\mathrm{CNN}) \# 3$ and $\mathrm{CNN} \# 5$ structures. The dots along the "reshaped to vector" line denote elements of the convolutional core output reshaped to a vector, which is the fully-connected classifier input data. 


\subsection{Proposed DCNN Architectures}

Six DCNNs that we have constructed are able to perform binary classification on satellite mosaics data (IR alone or IR + WV) represented as grayscale $100 \times 100$ pixels images:

1. CNN \#1. This model is built "from scratch" which means we have not used any pre-trained networks. CNN \#1 is built in the manner proposed in [35]. We varied sizes of convolutional kernels of each convolutional layers from $3 \times 3$ to $5 \times 5$. We also varied sizes of subsampling layers' receptive fields from $2 \times 2$ to $3 \times 3$. For each convolutional layer, we varied the number of convolutional kernels: $8,16,32,64$ and 100. The network convolutional core consists of three convolutional layers alternated with subsampling layers. Each pair of convolutional and subsampling layers is followed by a dropout layer. CNN\#1 is one-branched, and objects are described by IR $500 \times 500 \mathrm{~km}$ satellite snapshots only.

2. CNN \#2. This model is built "from scratch" with two separate branches-for IR and WV data. The convolutional core of each branch is built in the same manner as the convolutional core for $\mathrm{CNN} \# 1$ and as proposed in [41]. We varied the same parameters of the structure here in the same ranges as for $\mathrm{CNN} \# 1$.

3. CNN \#3. This model is built with TL approach. We used VGG16 pre-trained convolutional core to construct this model. None of VGG16 weights were optimized within this model, and only the weights of the FC classifier were trainable. This model is one-branched, and the objects are described by IR $500 \times 500 \mathrm{~km}$ satellite snapshots only. CNN \#3 structure is shown in Figure 3 .

4. CNN \#4. This model is two-branched, and each branch of its convolutional core is built with TL approach, in the same manner as the convolutional core of CNN \#3. Input data are IR and WV. None of VGG16 weights of this model in any of the two branches were optimized, and only the weights of the FC classifier were trainable. CNN \#4 structure is shown in Figure 4.

5. CNN \#5 is built with both TL and FT approaches. We built the convolutional core of this model with the use of VGG16 pre-trained network. VGG16 convolutional core consists of five similar blocks of layers. For the $\mathrm{CNN} \# 5$ we turned the last of these five blocks to be trainable. This model is one-branched, and objects are IR $500 \times 500 \mathrm{~km}$ satellite snapshots only. CNN \#5 structure is shown in Figure 3.

6. $\mathrm{CNN} \# 6$ is two-branched, and branches of its convolutional core are built in the same manner as the convolutional core of $\mathrm{CNN} \# 5$. For the $\mathrm{CNN} \# 6$, we turned the last of five blocks of each VGG16 convolutional cores to be trainable. Input data are IR and WV $500 \times 500 \mathrm{~km}$ satellite snapshots of dataset samples. CNN \#6 structure is shown in Figure 4.

\subsection{Computational Experiment Design}

The following hyper-parameters are included in each of the six networks:

- $\quad$ Size (number of nodes) of the first layer of FC classifier (denoted as FC1 in Figures 3 and 4)

- Convolutional kernels count for each convolutional layer (only applies to CNN \#1 and CNN \#2)

- Sizes of convolutional kernels (only applies to CNN \#1 and CNN \#2)

- Sizes of receptive fields of subsampling layers (only applies to CNN \#1 and CNN \#2)

The whole dataset was split into training (8952 samples) and testing (2237 samples) sets stratified by target value meaning that each set has the same (55:45) ratio of true/false samples as the whole dataset (i.e., 4924:4028 and 1253:984 samples in training and testing sets correspondingly). We have conducted hyper-parameters optimization for each of these DCNNs using stratified K-fold (K = 5) cross-validation approach. After this optimization, we trained several (typically 14-18) models with the best hyper-parameters configuration on the training set for each architecture (architecture-specific models). Then, we excluded models with the maximal and minimal accuracy score being estimated with the cross-validation approach, from this set of architecture-specific models for each of the six architectures. The remaining architecture-specific models were evaluated on the testing set, which was 
never seen by these models. We estimated the accuracy score for each individual model and the variance of accuracy score for the particular architecture with the best hyper-parameters combination (see Table 2).

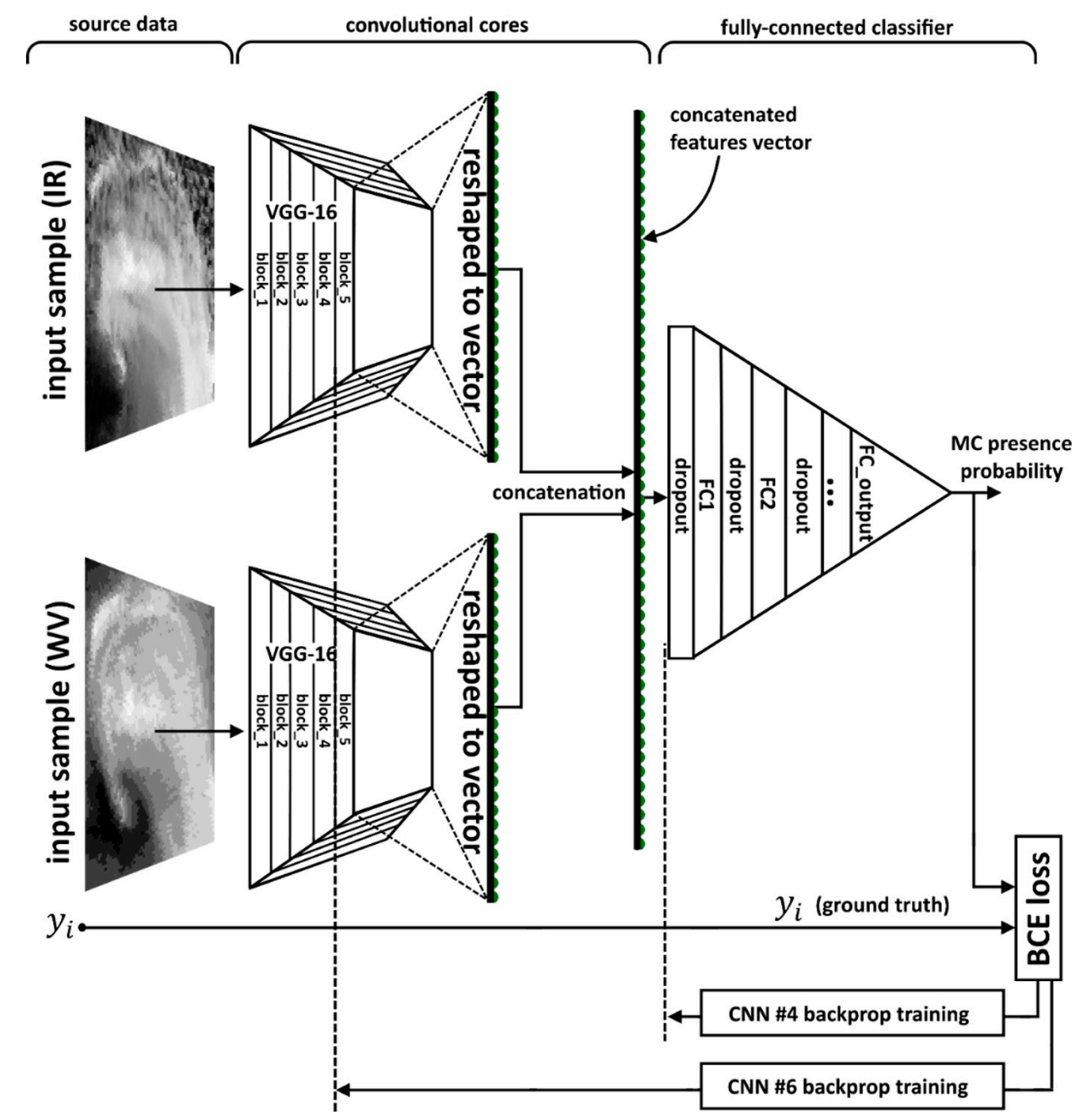

Figure 4. CNN \#4 and CNN \#6 structures. The dots along the "reshaped to vector" and "concatenated features vector" lines denote elements of convolutional cores outputs reshaped to vectors, which are, being concatenated to a combined features vector, the fully-connected classifier input data.

With the ensemble averaging approach, we evaluated the second-order models on the "never-seen by the model" testing set. As described in Section 3.3, we estimated the optimal probability threshold $p_{t h}$ for each second-order model and for the third-order model (see Table 2) for the best accuracy score estimation. These scores are treated as the quality measure of each particular architecture.

Numerical optimization and evaluation of models were performed at the Data Center of Far Eastern Branch of Russian Academy of Sciences (FEB RAS) [67] and DL computational resources of Sea-Air Interactions Laboratory of Shirshov Institute of Oceanology of Russian Academy of Sciences (IORAS, https://sail.ocean.ru/). Exploited computational nodes contain two graphics processing units (GPU) NVIDIA Tesla P100 16GB RAM. With these resources, the total GPU time of calculations is $3792 \mathrm{~h}$.

\section{Results}

The designed DCNNs were applied to detect of Antarctic MCs for the period from June to September 2004. Summary of the results of the application of six models is presented in Table 2. As we noted above, each model is characterized by the utilized data source (IR alone or IR + WV, 
columns "IR" and "WV" in Table 2). These DCNNs are further categorized according to a chosen set of applied techniques in addition to the basic approach (see Table 2 legend). Table 2 also provides accuracy scores and probability thresholds estimated, as described in Section 3.5, for the individual, second-, and third-order models of each architecture.

Table 2. Accuracy score of each model with the best hyper-parameters combination. BA-basic approach [41], TL-Transfer Learning, FT-Fine Tuning, Do-dropout, DA—dataset augmentation. Acc is the accuracy score averaged across models of the particular architecture. AsEA is the accuracy score of the ensemble averaged models with the optimal probability threshold. $p_{t h}$ is the optimal probability threshold value.

\begin{tabular}{|c|c|c|c|c|c|c|c|c|c|c|}
\hline $\begin{array}{l}\text { Model } \\
\text { Name }\end{array}$ & IR & WV & BA & TL & FT & Do & DA & $A c c$ & AsEA & $p_{t h}$ \\
\hline CNN \#1 & $X$ & - & $X$ & - & - & $x$ & $X$ & $86.89 \pm 1.1 \%$ & $89.3 \%$ & 0.381 \\
\hline CNN \#2 & $X$ & $X$ & $X$ & - & - & $x$ & $X$ & $94.1 \pm 1.4 \%$ & $96.3 \%$ & 0.272 \\
\hline CNN \#3 & $X$ & - & $X$ & $x$ & - & $X$ & $\mathrm{X}$ & $95.8 \pm 0.1 \%$ & $96.6 \%$ & 0.556 \\
\hline CNN \#4 & $X$ & $x$ & $X$ & $x$ & - & $x$ & $X$ & $95.5 \pm 0.3 \%$ & $96.3 \%$ & 0.526 \\
\hline CNN \#5 & $X$ & - & $X$ & $x$ & $X$ & $X$ & $X$ & $96 \pm 0.2 \%$ & $96.6 \%$ & 0.5715 \\
\hline CNN \#6 & $X$ & $X$ & $X$ & $X$ & $X$ & $X$ & $X$ & $95.7 \pm 0.2 \%$ & $96.4 \%$ & 0.656 \\
\hline \multicolumn{9}{|c|}{ Third-order model CNN \#1-6 averaged ensemble } & $97 \%$ & 0.598 \\
\hline
\end{tabular}

Figure 5 demonstrates four main types of false classified objects. The first and the second types are the ones for which IR data are missing completely or partially. The third type is the one for which the source satellite data were suspected to be corrupted. These three types of classifier errors originating from the lack of source data or the corruption of source data. For the fourth type, the source satellite data were realistic but the classifier has made a mistake. Thus, some of false classifications are model mistakes, and some are associated with the labeling issue where human expert could guess on the MC propagation over the area with missing or corrupted satellite data.

As shown in Table 2, CNN \#3 and CNN \#5 demonstrated the best accuracy among the second-order models on a never-seen subset of objects. The best combination of hyper-parameters for these networks is presented in Appendix B. Confusion matrices and receiver operating characteristic (ROC) curves for these models are shown in Figure 6a-d. Confusion matrices and ROC curves for all evaluated models are presented in Appendix C. Figure 6 clearly confirms that these two models perform almost equally for the true and the false samples. According to Table 2, the best accuracy score is reached using different probability thresholds for each second- or third-order model.

Comparison of CNN \#1, CNN \#2, on the one hand, and the remaining models, on the other hand, shows that DCNNs built with the use of TL technique demonstrate better performance when compared to the models built "from scratch". Moreover, the accuracy score variances of CNN \#1 and CNN \#2 are higher than for the other architectures. Thus, models built with TL approach seem to be more stable, and their generalization ability is better, as compared to models built "from scratch".

Comparing CNN \#1 and CNN \#2 qualities, we may conclude that the use of an additional data source (WV) results in the significant increase of the model accuracy score. Comparison of models within each pair of the network configurations (CNN \#3 vs. CNN \#5; CNN \#4 vs. CNN \#6) demonstrates that the FT approach does not provide significant improvement of the accuracy score in case of such a small size of the dataset. It is also obvious that the averaging over the ensemble members does increase the accuracy score from $0.6 \%$ for $\mathrm{CNN} \# 5$ to $2.41 \%$ for CNN \#1. However, in some cases, these score increases are comparable to the corresponding accuracy standard deviations.

It is also clear from the last row of Table 2, that the third-order model, which averages probabilities that are estimated by all trained models CNN \#1-6, produces the accuracy of $A c c=97 \%$ which outperforms all scores of individual models and second-order ensemble models. ROC curve and confusion matrices for this model are presented in Figure 6e,f. 
Figure 7 demonstrates the characteristics of the best model (third-order ensemble-averaging model) regarding false negatives (FN). Since the testing set is unbalanced with respect to stages, types of cyclogenesis and cloud vortex types, which we present in Figure 7a,c,d relative FN rates for each separate class in each taxonomy. We present the testing set distribution of classes for these taxonomies as well. Note that scales are different for reference distributions of classes of the testing set and the distributions of missed MCs. Detailed false negatives characteristics may be found in Appendix D.
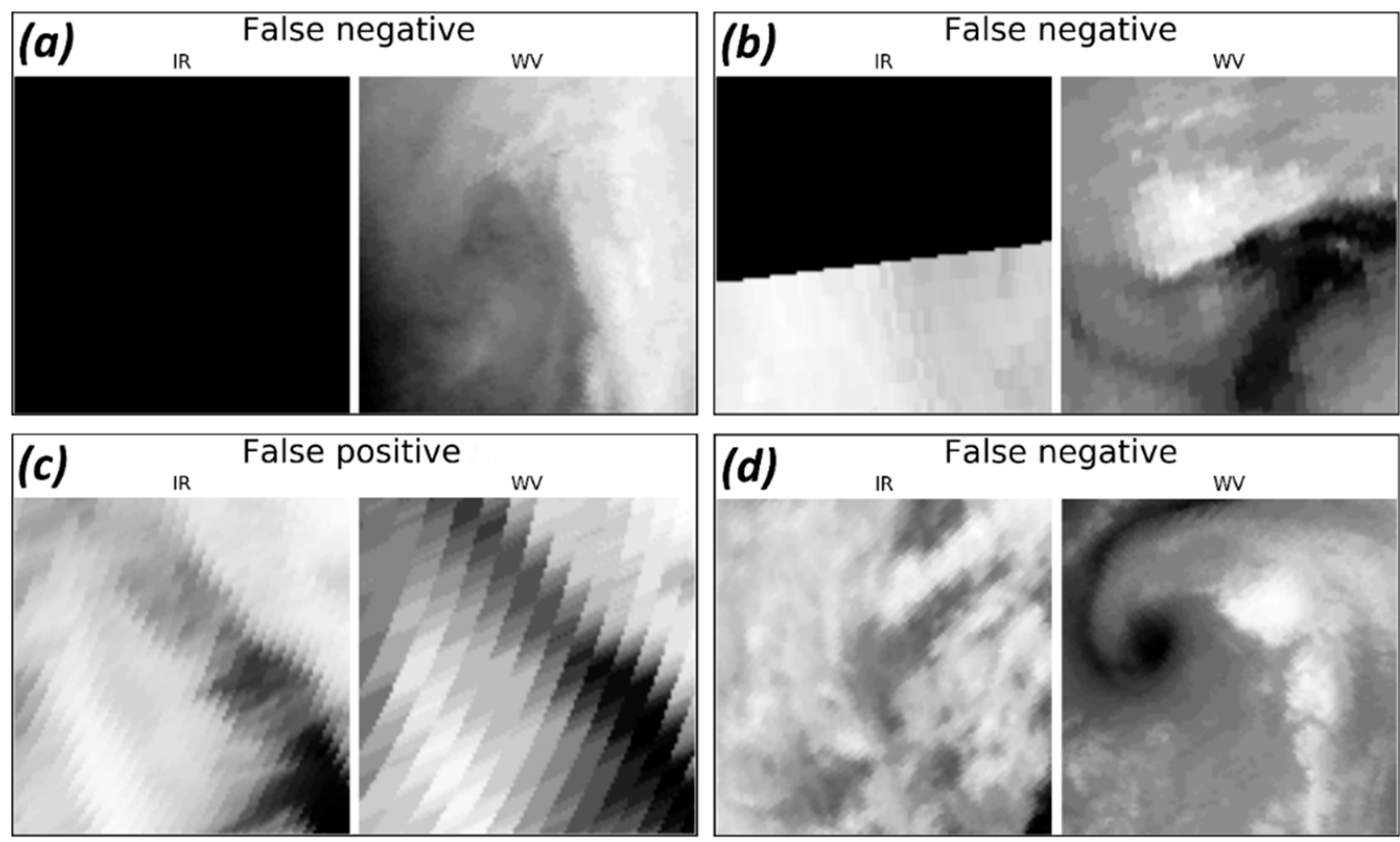

Figure 5. Examples of false classified objects, for which (a,b) IR satellite data is missing or corrupted, (c) the source satellite data is suspected to be corrupted, (d) the source satellite data is realistic, but the classifier has made a mistake.

Tracking procedure requires the sustainable ability of the MCs detection scheme to recognize mesocyclone cloud shape imprints during the whole MC life cycle. Figure 7a demonstrates that the best model classifies mesocyclone imprints almost equally for incipient ( $4.6 \%$ incipient missed) and mature ( $\sim \%$ mature missed) stages. The fraction of missed MCs in its dissipating stage is lower $(\sim 4 \%$ missed among MCs in dissipating stage). As for distribution of missed MCs with respect to their diameters (see Figure $7 \mathrm{~b}$ ), the histogram demonstrates fractions of FN objects relative to the whole FN number. The distribution of MC diameters in the testing set in Figure $7 \mathrm{~b}$ is shown as a reference. There is a peak around the diameter value of $325 \mathrm{~km}$, which does not coincide with any issues of distributions of MC diameters when the testing set is subset by any particular class of any taxonomy. However, since the total number of missed MCs is too small, there is no obvious reason to make assumptions on the origin of this issue. The FN rates per cyclogenesis types (Figure 7c) demonstrate the only issue for the orography-induced MCs. This issue is caused by the total number of that cyclogenesis type, which is small (only $27 \mathrm{MCs}$ in the testing set and only 134 in the training set), so the four that were missed is a substantial fraction of it. The same issue is demonstrated for the FN rates per cloud vortex types. Since the total number of "spiral cloud" type in the testing set is relatively small (59 of 1253), the five missed are a substantial fraction of it, as compared to 33 missed of 1006 for "comma cloud" type. 
(a)
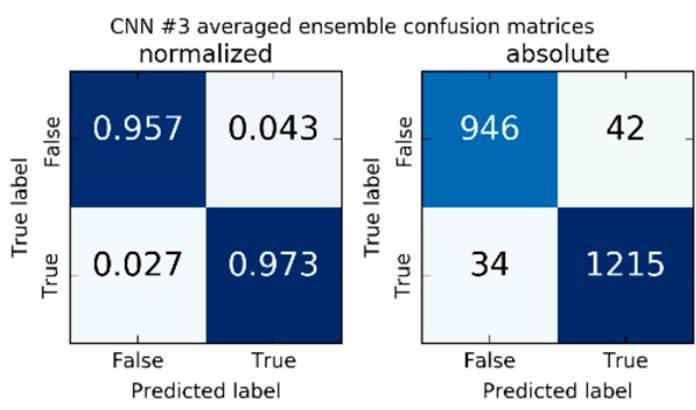

(c)
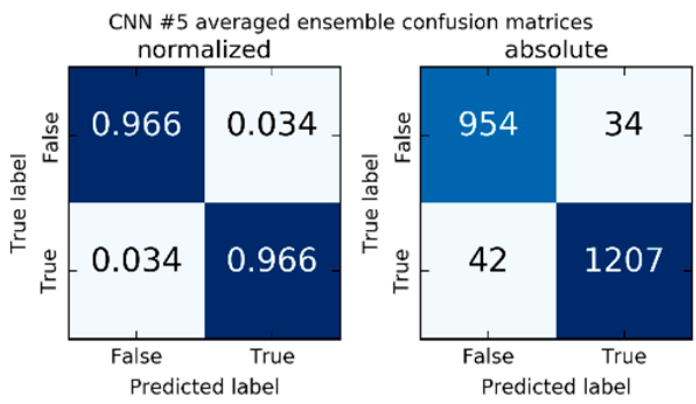

(e)
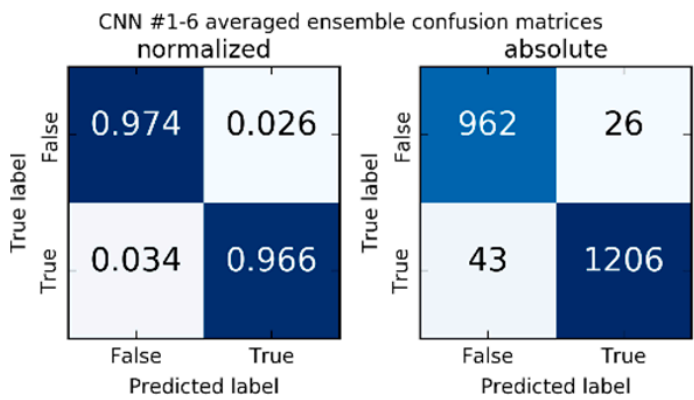

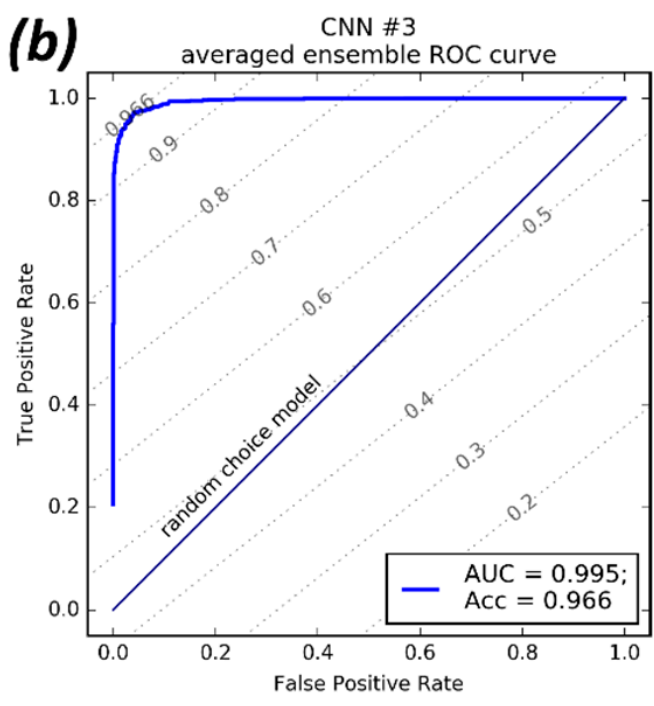

(d)

CNN \#5

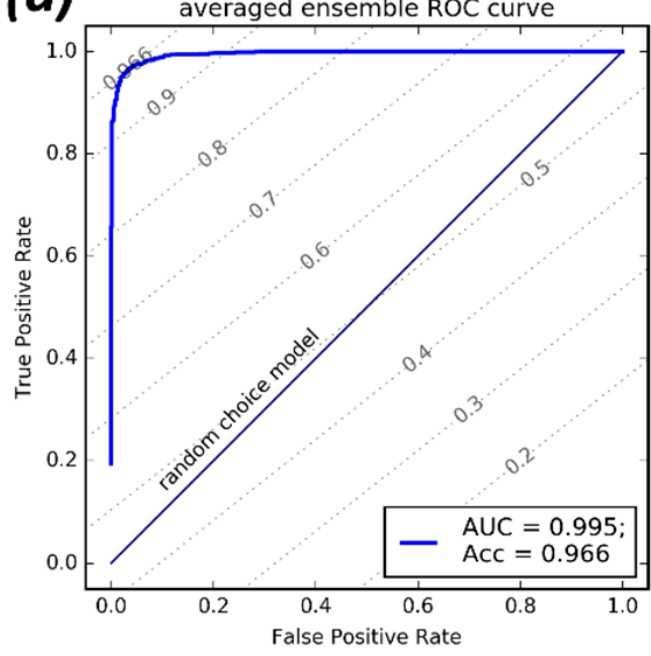

(f) CNN \#1-6

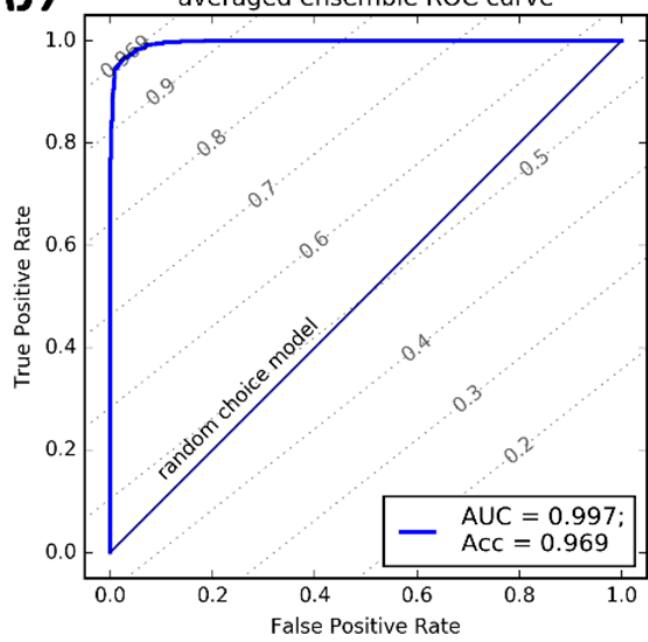

Figure 6. Confusion matrices and receiver operating characteristic curve for $(\mathbf{a}, \mathbf{b}) \mathrm{CNN} \# 3$ and (c,d) CNN \#5, both with the ensemble averaging approach applied (second-order models); and (e,f) third-order model CNN \#1-6 averaged ensemble. 

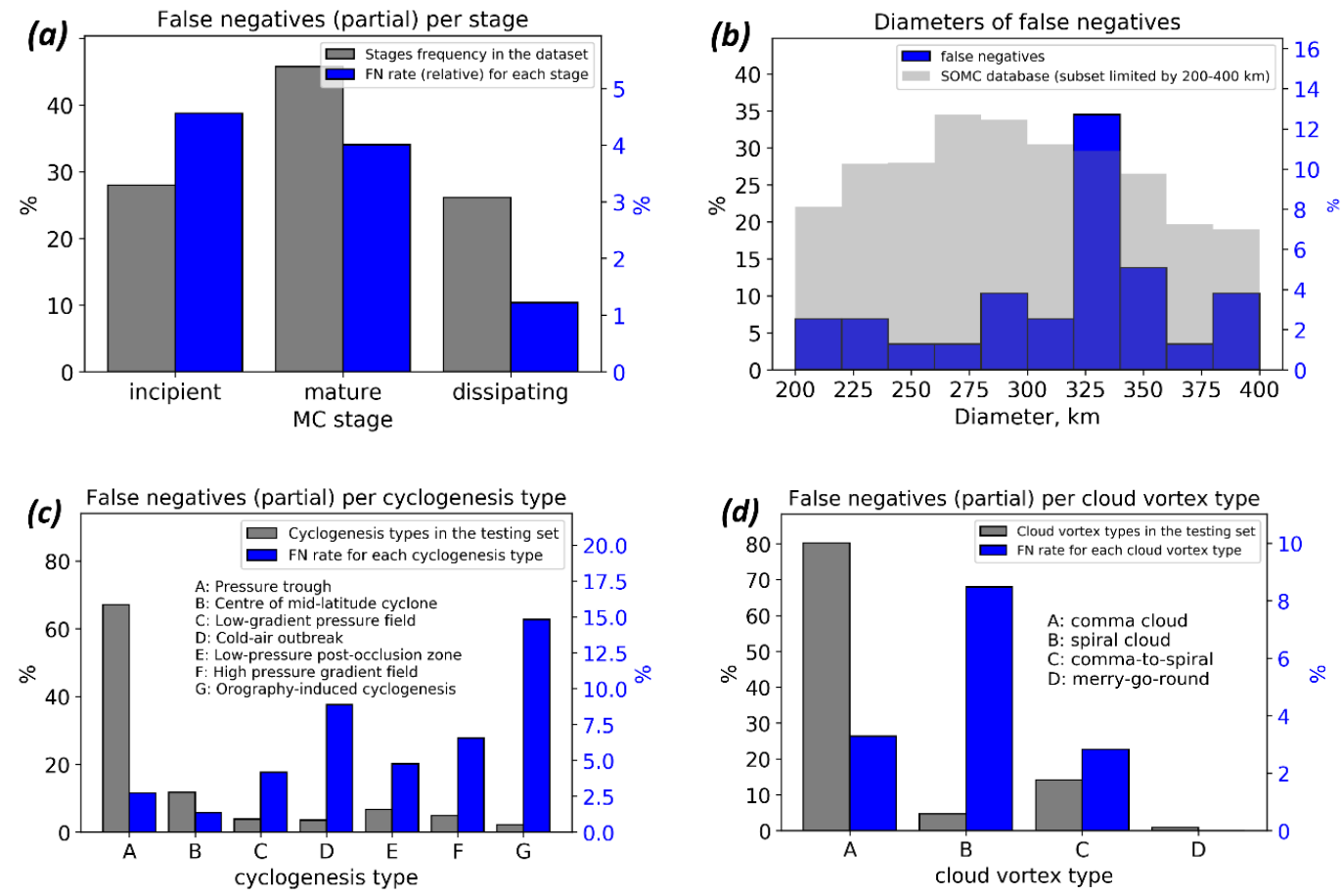

Figure 7. False negatives (FN, which are missed MCs) in the never-seen by the model testing set with respect to (a) lifecycle stages; (b) diameters; (c) cyclogenesis types; and, (d) types of cloud vortex.

\section{Conclusions and Outlook}

In this study, we present an adaptation of a DCNN method resulting in an algorithm that recognizes MCs signatures in preselected patches of satellite imagery of cloudiness and spatially collocated WV imagery. The DCNN technique shows very high accuracy in this problem. The best accuracy score of $97 \%$ is reached using the third-order ensemble-averaging model (six models ensemble) and the combination of both IR and WV images as input. We assess the accuracy of MCs recognition by comparison of identified MCs (true/false-image contain MC/no MC on the image parameter) with a reference dataset [5]. We demonstrate that deep convolutional networks are capable of effectively detecting the presence of polar mesocyclone signatures in satellite imagery patches of size $500 \times 500 \mathrm{~km}$. We also conclude that the quality of the satellite mosaics is sufficient enough for performing the task of binary classification regarding the MCs presence in $500 \times 500 \mathrm{~km}$ patches, and for performing other similar tasks of pattern recognition type, e.g., semantic segmentation of MCs.

Since the satellite-based studies of polar mesocyclone activity conducted in the $\mathrm{SH}$ (and in $\mathrm{NH}$ as well) have never reported season-dependent variations of IR imprint of cloud shapes of MCs $[23,27,68,69]$, we assume the proposed methodology to be applicable to satellite imageries of polar MCs that are available for the whole satellite observation era in $\mathrm{SH}$. In the $\mathrm{NH}$, the direct application of the models that were trained on $\mathrm{SH}$ dataset is restricted due to the opposite sign of relative vorticity, and thus, different cloud shape orientation. However the proposed approach is still applicable, and the only need is a dataset of tracks of MCs from the NH.

It was also shown that the accuracy of MCs detection by DCNNs is sensitive to the single (IR only) or double $(\mathrm{IR}+\mathrm{WV})$ input data usage. IR $+\mathrm{WV}$ combination provides significant improvement of the detection of MCs and allows a weak DCNN (CNN \#2) to detect MCs with higher accuracy compared to the weak CNN \#1 (89.3\% and $96.3 \%$ correspondingly). The computational cost of DCNN training and hyper-parameters optimization for deep neural networks are time- and computational-consuming. However, once trained, the computational cost of the DCNN inference is low. Furthermore, the trained DCNN performs much faster when compared to a human expert. Another advantage of the proposed method is the low computational cost of data preprocessing that allows the processing of satellite imagery in real time or the processing of large amounts of collected satellite data. 
We plan to extend the usage of this set of DCNNs (Table 2) for the development of an MCs tracking method based on machine learning and using satellite IR and WV mosaics. These efforts would be mainly focused on the development of the optimal choice of the "cut-off" window that has to be applied to the satellite mosaic. In the case of a sliding-window approach (e.g., running the $500 \times 500 \mathrm{~km}$ sliding window through the mosaics), the virtual testing dataset of the whole mosaic is highly unbalanced, so a model with non-zero FPR evaluated on balanced dataset would produce much higher FPR. Thus, we expect the sliding-window approach not to be accurate enough in the problem of MC detection. In the future, instead of the sliding-window, the Unet-like $[70,71]$ architecture should be considered with the binary semantic segmentation problem formulation. Since the models that have been applied in this study (specifically their convolutional cores) are capable of extracting the hidden representation that is relevant to MCs signatures, they may be used as the encoder part of the Unet-like encoder-decoder neural network for MCs identification and tracking. Considering MC tracking development, an approach proposed in a number of face recognition studies should be reassuring [72,73]. This approach can be applied in a manner of triple-based training of the DCNN to estimate a measure of similarity between one particular MC signatures in consecutive satellite mosaics.

Author Contributions: Conceptualization, M.K., P.V. and S.G.; Data curation, M.K. and M.L.; Formal analysis, M.K.; Funding acquisition, S.G.; Investigation, M.K. and K.G.; Methodology, M.K. and P.V.; Project administration, M.K.; Resources, P.V. and S.G.; Software, M.K. and K.G.; Supervision, S.G.; Validation, M.K., P.V. and S.G.; Visualization, M.K. and P.V.; Writing-original draft, M.K., P.V., N.T. and M.L.; Writing-review \& editing, N.T., S.G. and M.L.

Funding: This research was funded by the Russian Ministry of Education and Science (agreement 14.613.21.0083, project ID RFMEFI61317X0083). Materials from MAL are based upon the work funded by the United States National Science Foundation under grants ANT-1244924 and ANT-1535632.

Acknowledgments: Computational resources for this research were provided by the Shared Facility Center "Data Center of FEB RAS", Khabarovsk, Russia.

Conflicts of Interest: The authors declare no conflict of interest. The funders had no role in the design of the study; in the collection, analyses, or interpretation of data; in the writing of the manuscript, and in the decision to publish the results.

\section{Nomenclature}

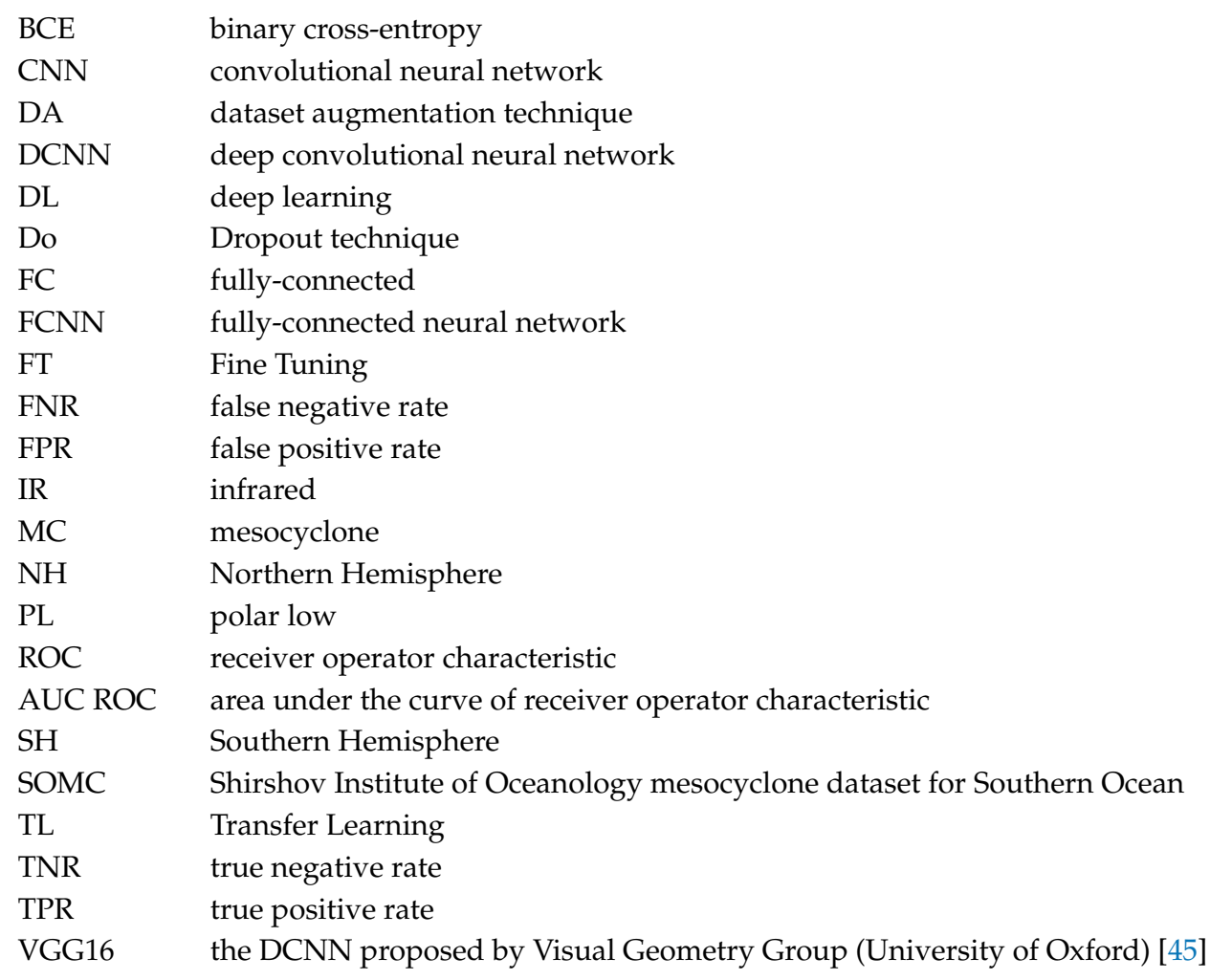




\section{Appendix A. DCNN Best Practices and Additional Techniques}

There is a set of best practices commonly used to construct DCNNs for solving classification problems [56]. Modern DCNNs are built on the basis of consecutive convolutional and subsampling layers by performing nonlinear transformation of the initial data (see Figure 2 in [41]). The primary layer type of convolutional neural networks (CNNs) is the so-called convolutional layer which is designed to extract visual patterns density map using discrete convolution operation with $K$ (tends to be from 3 to 1000) kernels followed by a nonlinear transformation operation (activation function). One additional layer type is a pooling layer performing subsampling operation with one of the following aggregation functions: maximum, minimum, mean or others. In the current practice the maximum is used.

Since the LeNet DCNN [41] several studies [41-44] have demonstrated that the usage of consecutive convolutional and subsampling layers results in a skillful detection of various spatial patterns from the input 2D sample. The approach proposed in [41] implies the use of the output of these stacked layers set as an input data for a classifier, which in general may be any method suitable for classification problems, such as linear models, logistic regression, etc. LeCun [41] suggested to use the neural classifier, and this is now a conventional approach. The advantage of using a neural classifier is the ability to train the whole model at once (the so-called end-to-end training).

The whole model built in this manner represents a classifier capable of direct predicting a target value for the sample. We term the fully-connected (FC) layers set as "FC classifier", and the preceding part containing convolutional and pooling layers as "convolutional core" (see Figures 3 and 4).

For building a DCNN it is important to account for data dimensionality during its transformations from layer to layer. The input for a DCNN is an image represented by a matrix of the size $(h, w, d)$, where $h$ and $w$ correspond to the image height and width in pixels, $d$ is its levels number, the so-called depth (e.g., $d=3$ when levels are red, green and blue channels of a colorful image). For the water vapor or radio-brightness temperature satellite data, $d=1$. A convolutional layer and subsampling layer are described in details in [41]. Convolutional layers are characterized by their kernel sizes (e.g., $3 \times 3,5 \times 5)$, their kernel numbers $K$ and the nonlinear operation used (e.g., tanh in [41]). Subsampling layers are characterized by their receptive field sizes e.g., $3 \times 3,5 \times 5$ etc. The output of a convolutional layer with $K$ kernels is the so-called feature maps which is a matrix of the size $(h, w, K)$. The nonlinear operation transforms it to a matrix of size $(h, w, 1)$. The following subsampling layer reduces the matrix size depending on the subsampling layer kernel size. Typically, this size is $(2,2)$ or $(3,3)$. Thus, the subsampling operation reduces the sample size by a factor 2 or 3 , respectively. The output of a convolutional core is a set of abstract feature maps which is represented by a 3D matrix. This matrix, being reshaped into a vector, is passed as the input to the FC classifier (see Figures 3 and 4).

FC classifier of all models of this study includes hidden FC layers whose count varied from 2 to 4. FC layers are characterized by the number of its basic building blocks (so-called artificial neurons), which transform input data according to their rules (activation function) and parameters (so-called weights) [53,74]. Artificial neurons count of FC1 which is the layer following the convolutional core (see Figures 3 and 4), is chosen from the set $\{128,256,512,1024\}$. The size of each following FC layer is half of the preceding one, but not less than 128. The output layer is fully-connected as well and contains one output node. For example, the structure of FC classifier in terms of nodes count of layers might be the following: $\{512 ; 256 ; 128 ; 1\}$. All FC layers are alternated with dropout layers in order to prevent overfitting of the model. All trainable layers' activation functions are Rectified Linear Unit (ReLU):

$$
\sigma_{R e L U}(z)=\max (0 ; z)
$$

except the output layer whose activation function is sigmoid:

$$
\sigma_{\text {sigm }}(z)=\frac{1}{1+e^{-\theta z}}
$$

where $\theta$ are layers' trainable parameters.

In order to measure the error of the network on each individual sample during the training process we use the binary cross-entropy as a loss function:

$$
\mathcal{L}=\sum_{i=0}^{N}\left(y_{i} \log \hat{y}_{i}+\left(1-y_{i}\right) \log \left(1-\hat{y}_{i}\right)\right)
$$

where $y_{i}$ is the expert-defined ground truth for the target value, $\hat{y}_{i}$ is the estimated probability of the $i$-th sample to be true, $N$ is samples count of the training set or a training mini-batch. This loss 
function is minimized in the space of the model weights using the method of backpropagation of error [66] denoted as "backprop training" in Figures 3 and 4. The outcome of the the whole model is the probability of each class for the input sample. In the case of binary classification, the FC classifier has one output unit, producing probability of MC presence for the input sample.

In addition to the basic approach proposed in [41] a number of techniques may be applied. Using them one can construct and train DCNNs of various accuracy and various generalization abilities which is characterized by the quality of a model estimated on a never-seen test data.

\section{Appendix A.1. Transfer Learning}

One of the additional approaches is Transfer Learning (TL) [57-62]. Generally, this technique focuses on storing the knowledge obtained by some network while being trained for one problem and applying it to another problem of a similar kind. In practice, this approach implies the DCNN structure to be built using some part of a network previously trained on a considerable amount of data, for example, ImageNet [49]. In these terms, VGG16 [45] is not only an efficient architecture, but also the pre-trained network containing optimized weights values (also known as network parameters). Best practice for building a new advanced DCNN based on TL approach is to compose it using convolutional core of the pre-trained model (e.g., VGG16) followed by a new FC neural classifier. Weights of the convolutional part in this case are fixed, and only FC part is optimized. In this approach, the convolutional core may be considered as a feature extractor (see [41]), which computes a highly relevant low-dimensional (compared to original samples dimensionality) vector, representing the data (e.g., "reshaped to vector" output of the convolutional core in Figure 3).

\section{Appendix A.2. Fine Tuning}

TL approach relies on the similarity of data distributions within two datasets. But in the case of significant differences, for example in terms of Kullback-Leibler divergence between some particular feature approximated probability distributions, the new FC classifier capabilities may not cover all of those differences. In this case, some layers of the convolutional core, that are close to FC classifier, can be turned on to be optimized (the so-called Fine Tuning, FT). Regarding DCNNs application to satellite mosaics, we have to consider that VGG16 was optimized on ImageNet dataset which contains everyday-observed objects like buildings, dogs, cats, cars etc., without any satellite imageries or even clouds. So FT approach can be considered as a promising approach when composing MC-detecting DCNN at IR and WV satellite mosaic data.

\section{Appendix A.3. Preventing Overfitting}

Machine learning models and neural networks, in particular, may vary in terms of complexity. Overly complex models may suffer from overfitting: the effect of poor target prediction quality on unseen data concurrently with nearly exact prediction of target values on training data. There are several state-of-the-art approaches to prevent overfitting of neural networks. We used most fruitful and reliable ones: dropout [64] and data augmentation also called auxiliary variables [65]. We also used ensemble averaging of the models outcome.

\section{Appendix A.4. Preventing Overfitting with Dropout}

Dropout approach is the way of preventing overfit with a computationally inexpensive but still powerful method of regularizing neural networks through bagging [75] and virtually ensembling models of similar architecture. Bagging involves training multiple models and testing each of them on test samples. Since training and evaluating of deep neural networks tend to be time-consuming and computationally expensive, the original bagging approach [75] seems to be impractical. With the dropout approach applied, the network may be thought of as an ensemble of all sub-networks that can be composed by removing non-output nodes from the base network. In practice, this approach is implemented by dropout layer which turns the preceding layer output to zero for each node with some probability $p$. This procedure repeats for each mini-batch at the training time. At the inference time, the dropout approach involves network weights scaling by $1 / p$. Each of our models includes dropout layers between trainable layers. Rate $p$ was set to 0.1 for each dropout layer of each model.

\section{Appendix A.5. Preventing Overfitting with Dataset Augmentation}

Dataset augmentation is the state-of-the-art way to make a machine learning model generalize better. When available dataset size is limited, the way to get around that is to generate fake data which 
should be similar to real samples. Best practice for DCNNs is generating fake samples by adding some noise or applying slight transformations like shift, shear, rotation, scaling etc. Formally, with data augmentation one can increase variability of features of the original dataset and substantially extend its size. This approach often improves generalization ability of the trained model.

We trained each of our models with data augmentation approach applied. The rotation angle range was $90^{\circ}$ in both direction; independent width and height scaling performed within range from 0.8 to 1.2; zoom range from 0.8 to 1.2; shear angle range from $-2^{\circ}$ to $2^{\circ}$. We did not use flipping upside-down and left-to-right.

\section{Appendix A.6. Preventing Overfitting with Ensemble Averaging}

In general, during the parameters optimization (learning process) each DCNN converges to a local minimum of the loss function in the space of its weights. The training process starts from a randomly generated point of this space. Due to a non-convexity of loss function, every new DCNN model converges to a new local minimum. Some models may converge to a minimum that is not really close to a global one in terms of loss function value, and thus the quality measure of that model remains poor. Other models may converge to a good minimum that is close to a global one in terms of loss function value, but this proximity may lead to a poor generalization ability which means low quality measure estimated on a testing subset of data. There are approaches for improving the generalization ability of several models that are generally similar, but differ in detailed predictions. In our study we applied simple ensemble averaging [76], which is one of state-of-the-art approaches for improving machine learning models generalization ability. With this approach several models of each architecture are trained, and probabilities of these models are averaged. The prediction of this model is treated as an ensemble outcome:

$$
p_{i}=\frac{\sum_{m=0}^{M} p_{i}^{(m)}}{M}
$$

where $p_{i}$ is the estimated probability of the ensemble of $M$ models for $i$-th sample to be true; each $m$-th model's probability estimation for $i$-th sample to be true is $p_{i}^{(m)}$. In this study we applied ensembling on DCNNs of identical architectures. The resulting models we term second-order models in this study. They are synthetic ones that are not trained, but are ensembles.

Satellite IR + WV snapshots or satellite IR snapshot alone are essentially the object description, and each model that is presented in our study produces the outcome for each object regardless of the description-whether it is IR snapshot alone or IR + WV snapshots. So there is an opportunity to average probability outcomes of all the models of this study. The resulting model that produces averaged probabilities of the ensemble containing all trained models we term third-order model. It is a synthetic one that is not trained, but is an ensemble.

\section{Appendix A.7. Adjustment of the Probability Threshold}

The outcome of each model of this study is the estimation of the probability for the sample to be true (i.e., to contain an MC). So there is an arbitrariness in choosing the threshold of this probability to get the outcome which is binary. The most common way to choose this threshold is the ROC curve analysis. Each point of this curve represents the False Positive Rate (FPR) and True Positive Rate (TPR) combination for the particular probability threshold $p_{t h}$ (e.g., see Figure $\left.6 \mathrm{~b}, \mathrm{~d}, \mathrm{f}\right)$. The model performing true random choice between true and false outcome has a ROC curve on the main diagonal of this plot. The ROC curve of the perfect classifier follows from the point $(0.0,0.0)$ straight to the point $(0.0,1.0)$ and then to the point $(1.0,1.0)$. The area under the ROC curve (AUC ROC) may be considered as a measure of model quality. The best model AUC ROC is 1.0, the true random choice model AUC ROC is 0.5 , and the worst model AUC ROC is 0.0 .

In a range of cases the best accuracy score might not be reached with $p_{t h}=0.5$. The lines of equal accuracy score, as presented in Figure $6 \mathrm{~b}, \mathrm{~d}, \mathrm{f}$, are diagonal. In case of perfect 50/50 ratio of true/false samples they are parallel to the main diagonal. In case of slight inequality of true and false samples count these lines have slightly different slope as shown in Figure $6 \mathrm{~b}$,d,f. For each accuracy score there are two, one or no points of the ROC curve intersection with the accuracy isoline. So if a model is represented with a ROC curve, the maximum value of its Acc is located at the point of this curve where the accuracy isoline is tangent to it. For each model of this study including second- and third-order models, the optimal probability threshold was estimated based on ROC curve analysis. 


\section{Appendix B. CNN \#3 and CNN \#5 Best Hyper-Parameters Combinations}

According to Section 3.4, CNN \#3 and CNN \#5 are both constructed to have one-branched convolutional core. Best combination of hyper-parameters of these networks are the same. The only difference is the FT approach that was applied in case of CNN \#5.

Table A1. CNN \#3 and CNN \#5 best hyper-parameters combination.

\begin{tabular}{ccc}
\hline Layer (Block) Name & Layer (Block) Nodes Count or Output Dimensions & Connected to \\
\hline Input_data_IR & $100 \times 100$ & - \\
VGG_16_conv_core & see [45]; output: $3 \times 3 \times 512$ & Input_data_IR \\
Reshape_1 & 4608 & VGG_16_conv_core \\
Dropout_1 & 4608 & Reshape_1 \\
FC1 & 1024 & Dropout_1 \\
Dropout_2 & 1024 & FC1 \\
FC2 & 512 & Dropout_2 \\
Dropout_3 & 512 & FC2 \\
FC3 & 256 & Dropout_3 \\
Dropout_4 & 256 & FC3 \\
FC4 & 128 & Dropout_4 \\
FC_output & 1 & FC3 \\
\hline
\end{tabular}

\section{Appendix C. Detailed Performance Metrics of all DCNN Models}
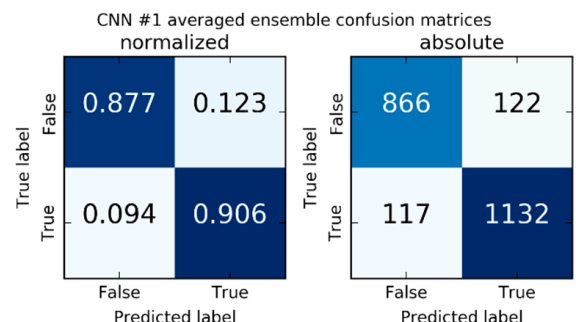

CNN \#3 averaged ensemble confusion matrices
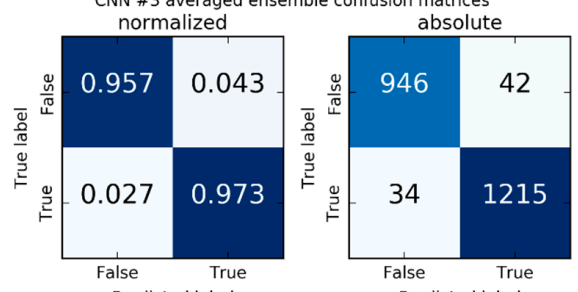

Predicted label
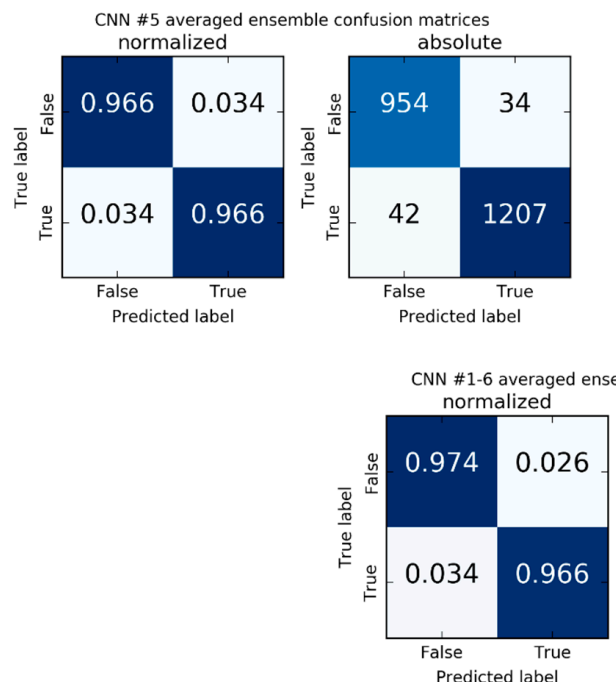

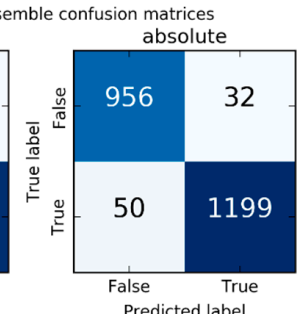

CNN \#4 averaged ensemble confusion matrices
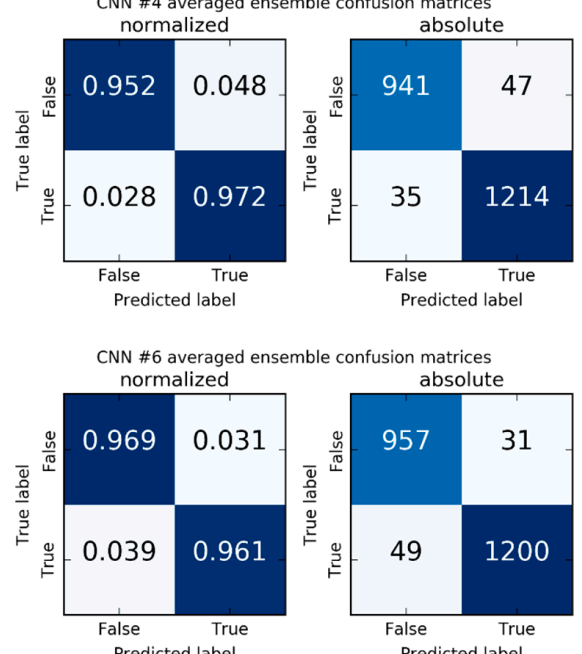

Figure A1. Confusion matrices for all models and the third-order model CNN \#1-6 averaged ensemble, computed on test never-seen subset of data. For each architecture the ensemble averaging technique is applied. 


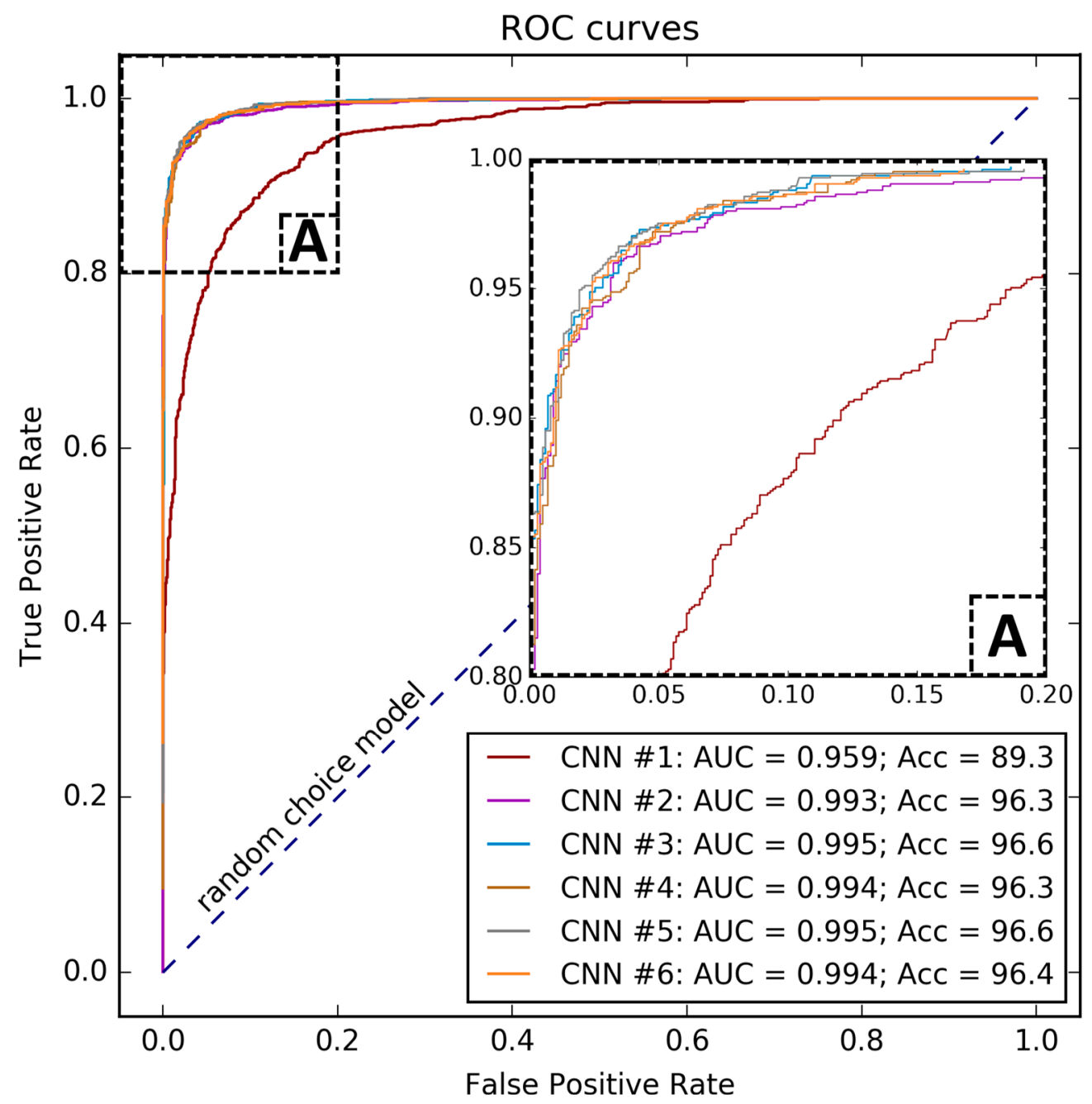

Figure A2. Receiver operating characteristic curves computed on test never-seen subset of data for all models. For each architecture the ensemble averaging technique is applied.

Appendix D. Detailed False Negative Rates of the Third-Order Ensemble-Averaging Model

Table A2. False negative rates per cyclogenesis types.

\begin{tabular}{cccc}
\hline Cyclogenesis Type & $\begin{array}{c}\text { Testing Set, } \\
\text { Objects Number }\end{array}$ & $\begin{array}{c}\text { False Negatives, } \\
\text { Objects Number }\end{array}$ & FN Relative Rate, \% \\
\hline Pressure trough & 841 & 23 & 2.7 \\
Centre of mid-latitude cyclone & 147 & 2 & 1.4 \\
Low-gradient pressure field & 48 & 2 & 4.2 \\
Cold-air outbreak & 45 & 4 & 8.9 \\
Low-pressure post-occlusion zone & 84 & 4 & 4.8 \\
High pressure gradient field & 61 & 4 & 6.6 \\
Orography-induced cyclogenesis & 27 & 4 & 14.8 \\
\hline
\end{tabular}

Table A3. False negative rates per cloud vortex types.

\begin{tabular}{cccc}
\hline Cloud Vortex Type & $\begin{array}{c}\text { Testing Set, } \\
\text { Objects Number }\end{array}$ & $\begin{array}{c}\text { False Negatives, } \\
\text { Objects Number }\end{array}$ & FN Relative Rate, \% \\
\hline Comma cloud & 1006 & 33 & 3.3 \\
Spiral cloud & 59 & 5 & 8.5 \\
Comma-to-spiral & 177 & 5 & 2.3 \\
Merry-go-round & 11 & 0 & 0.0 \\
\hline
\end{tabular}


Table A4. False negative rates per MC stages.

\begin{tabular}{cccc}
\hline MC Stage & $\begin{array}{c}\text { Testing Set, } \\
\text { Objects Number }\end{array}$ & $\begin{array}{c}\text { False Negatives, } \\
\text { Objects Number }\end{array}$ & FN Relative Rate, \% \\
\hline Incipient & 352 & 16 & 4.6 \\
Mature & 574 & 23 & 4.0 \\
Dissipating & 327 & 4 & 1.2 \\
\hline
\end{tabular}

\section{References}

1. Thorpe, A.J. Polar Lows: Mesoscale Weather Systems in the Polar Regions; Rasmussen, E.A., Turner, J., Eds.; Cambridge University Press: Cambridge, UK, 2003; ISBN 978-0-511-52497-4.

2. Marshall, J.; Schott, F. Open-ocean convection: Observations, theory, and models. Rev. Geophys. 1999, 37, 1-64. [CrossRef]

3. Condron, A.; Renfrew, I.A. The impact of polar mesoscale storms on northeast Atlantic Ocean circulation. Nat. Geosci. 2013, 6, 34-37. [CrossRef]

4. Condron, A.; Bigg, G.R.; Renfrew, I.A. Modeling the impact of polar mesocyclones on ocean circulation. J. Geophys. Res. Oceans 2008, 113. [CrossRef]

5. Verezemskaya, P.; Tilinina, N.; Gulev, S.; Renfrew, I.A.; Lazzara, M. Southern Ocean mesocyclones and polar lows from manually tracked satellite mosaics. Geophys. Res. Lett. 2017, 44, 7985-7993. [CrossRef]

6. Laffineur, T.; Claud, C.; Chaboureau, J.-P.; Noer, G. Polar lows over the Nordic Seas: Improved Representation in ERA-Interim Compared to ERA-40 and the Impact on Downscaled Simulations. Mon. Weather Rev. 2014, 142, 2271-2289. [CrossRef]

7. Michel, C.; Terpstra, A.; Spengler, T. Polar Mesoscale Cyclone Climatology for the Nordic Seas Based on ERA-Interim. J. Clim. 2017, 31, 2511-2532. [CrossRef]

8. Bromwich, D.H.; Wilson, A.B.; Bai, L.-S.; Moore, G.W.K.; Bauer, P. A comparison of the regional Arctic System Reanalysis and the global ERA-Interim Reanalysis for the Arctic. Q. J. R. Meteorol. Soc. 2016, 142, 644-658. [CrossRef]

9. Press, W.H.; Teukolsky, S.A.; Vetterling, W.T.; Flannery, B.P. Numerical Recipes 3rd Edition: The Art of Scientific Computing; Cambridge University Press: Cambridge, UK, 2007; ISBN 0-521-88068-8.

10. Rojo, M.; Claud, C.; Mallet, P.-E.; Noer, G.; Carleton, A.M.; Vicomte, M. Polar low tracks over the Nordic Seas: A 14-winter climatic analysis. Tellus A Dyn. Meteorol. Oceanogr. 2015, 67, 24660. [CrossRef]

11. Smirnova, J.; Golubkin, P. Comparing Polar Lows in Atmospheric Reanalyses: Arctic System Reanalysis versus ERA-Interim. Mon. Weather Rev. 2017, 145, 2375-2383. [CrossRef]

12. Hines, K.M.; Bromwich, D.H. Development and Testing of Polar Weather Research and Forecasting (WRF) Model. Part I: Greenland Ice Sheet Meteorology. Mon. Weather Rev. 2008, 136, 1971-1989. [CrossRef]

13. Zappa, G.; Shaffrey, L.; Hodges, K. Can Polar Lows be Objectively Identified and Tracked in the ECMWF Operational Analysis and the ERA-Interim Reanalysis? Mon. Weather Rev. 2014, 142, 2596-2608. [CrossRef]

14. Pezza, A.; Sadler, K.; Uotila, P.; Vihma, T.; Mesquita, M.D.S.; Reid, P. Southern Hemisphere strong polar mesoscale cyclones in high-resolution datasets. Clim. Dyn. 2016, 47, 1647-1660. [CrossRef]

15. Xia, L.; Zahn, M.; Hodges, K.; Feser, F.; Storch, H. A comparison of two identification and tracking methods for polar lows. Tellus A Dyn. Meteorol. Oceanogr. 2012, 64, 17196. [CrossRef]

16. Harold, J.M.; Bigg, G.R.; Turner, J. Mesocyclone activity over the North-East Atlantic. Part 1: Vortex distribution and variability. Int. J. Climatol. 1999, 19, 1187-1204. [CrossRef]

17. Noer, G.; Saetra, Ø.; Lien, T.; Gusdal, Y. A climatological study of polar lows in the Nordic Seas. Q. J. R. Meteorol. Soc. 2011, 137, 1762-1772. [CrossRef]

18. Smirnova, J.E.; Zabolotskikh, E.V.; Bobylev, L.P.; Chapron, B. Statistical characteristics of polar lows over the Nordic Seas based on satellite passive microwave data. Izv. Atmos. Ocean. Phys. 2016, 52, 1128-1136. [CrossRef]

19. McMurdie, L.A.; Claud, C.; Atakturk, S. Satellite-derived atmospheric characteristics of spiral and comma-shaped southern hemisphere mesocyclones. J. Geophys. Res. Atmos. 1997, 102, 13889-13905. [CrossRef] 
20. Irving, D.; Simmonds, I.; Keay, K. Mesoscale Cyclone Activity over the Ice-Free Southern Ocean: 1999-2008. J. Clim. 2010, 23, 5404-5420. [CrossRef]

21. Neu, U.; Akperov, M.G.; Bellenbaum, N.; Benestad, R.; Blender, R.; Caballero, R.; Cocozza, A.; Dacre, H.F.; Feng, Y.; Fraedrich, K.; et al. IMILAST: A Community Effort to Intercompare Extratropical Cyclone Detection and Tracking Algorithms. Bull. Am. Meteorol. Soc. 2013, 94, 529-547. [CrossRef]

22. Wilhelmsen, K. Climatological study of gale-producing polar lows near Norway. Tellus A Dyn. Meteorol. Oceanogr. 1985, 37, 451-459. [CrossRef]

23. Carrasco, J.F.; Bromwich, D.H. Mesoscale cyclogenesis dynamics over the southwestern Ross Sea, Antarctica. J. Geophys. Res. Atmos. 1993, 98, 12973-12995. [CrossRef]

24. Carrasco, J.F.; Bromwich, D.H.; Liu, Z. Mesoscale cyclone activity over Antarctica during 1991: 1. Marie Byrd Land. J. Geophys. Res. Atmos. 1997, 102, 13923-13937. [CrossRef]

25. Turner, J.; Thomas, J.P. Summer-season mesoscale cyclones in the bellingshausen-weddell region of the antarctic and links with the synoptic-scale environment. Int. J. Climatol. 1994, 14, 871-894. [CrossRef]

26. Harold, J.M.; Bigg, G.R.; Turner, J. Mesocyclone activity over the Northeast Atlantic. Part 2: An investigation of causal mechanisms. Int. J. Climatol. 1999, 19, 1283-1299. [CrossRef]

27. Carleton, A.M. On the interpretation and classification of mesoscale cyclones from satellite infrared imagery. Int. J. Remote Sens. 1995, 16, 2457-2485. [CrossRef]

28. Claud, C.; Katsaros, K.B.; Mognard, N.M.; Scott, N.A. Comparative satellite study of mesoscale disturbances in polar regions. Glob. Atmos. Ocean Syst. 1996, 4, 233-273.

29. Gang, F.; Qin-yu, L.; Zeng-mao, W. General features of polar lows over the Japan Sea and the Northwestern Pacific. Chin. J. Ocean. Limnol. 1999, 17, 300-307. [CrossRef]

30. Gurvich, I.A.; Pichugin, M.K. Study of the compara tive characteristics of typical mesoscale cyclones over Far Eastern seas on the basis of satellite multisensory sounding. Sovrem. Probl. Distantsionnogo Zondirovaniya Zemli Kosmosa 2013, 10, 51-59.

31. Claud, C.; Carleton, A.M.; Duchiron, B.; Terray, P. Southern hemisphere winter cold-air mesocyclones: Climatic environments and associations with teleconnections. Clim. Dyn. 2009, 33, 383-408. [CrossRef]

32. Blechschmidt, A.-M. A 2-year climatology of polar low events over the Nordic Seas from satellite remote sensing. Geophys. Res. Lett. 2008, 35. [CrossRef]

33. Heinle, A.; Macke, A.; Srivastav, A. Automatic cloud classification of whole sky images. Atmos. Meas. Tech. 2010, 3, 557-567. [CrossRef]

34. Taravat, A.; Frate, F.D.; Cornaro, C.; Vergari, S. Neural Networks and Support Vector Machine Algorithms for Automatic Cloud Classification of Whole-Sky Ground-Based Images. IEEE Geosci. Remote Sens. Lett. 2015, 12, 666-670. [CrossRef]

35. Onishi, R.; Sugiyama, D. Deep Convolutional Neural Network for Cloud Coverage Estimation from Snapshot Camera Images. SOLA 2017, 13, 235-239. [CrossRef]

36. Krizhevsky, A.; Sutskever, I.; Hinton, G.E. Imagenet classification with deep convolutional neural networks. In Advances in Neural Information Processing Systems; MIT Press: Cambridge, MA, USA, 2012; pp. 1097-1105.

37. Shin, H.-C.; Roth, H.R.; Gao, M.; Lu, L.; Xu, Z.; Nogues, I.; Yao, J.; Mollura, D.; Summers, R.M. Deep Convolutional Neural Networks for Computer-Aided Detection: CNN Architectures, Dataset Characteristics and Transfer Learning. IEEE Trans. Med. Imaging 2016, 35, 1285-1298. [CrossRef] [PubMed]

38. Krinitskiy, M.A. Application of machine learning methods to the solar disk state detection by all-sky images over the ocean. Oceanology 2017, 57, 265-269. [CrossRef]

39. Liu, Y.; Racah, E.; Correa, J.; Khosrowshahi, A.; Lavers, D.; Kunkel, K.; Wehner, M.; Collins, W. Application of deep convolutional neural networks for detecting extreme weather in climate datasets. arXiv, 2016; arXiv:1605.01156.

40. Huang, D.; Du, Y.; He, Q.; Song, W.; Liotta, A. DeepEddy: A simple deep architecture for mesoscale oceanic eddy detection in SAR images. In Proceedings of the 2017 IEEE 14th International Conference on Networking, Sensing and Control (ICNSC), Calabria, Italy, 16-18 May 2017; pp. 673-678.

41. Lecun, Y.; Bottou, L.; Bengio, Y.; Haffner, P. Gradient-based learning applied to document recognition. Proc. IEEE 1998, 86, 2278-2324. [CrossRef]

42. LeCun, Y.; Bengio, Y.; Hinton, G. Deep learning. Nature 2015, 521, 436-444. [CrossRef] [PubMed]

43. Liu, W.; Wang, Z.; Liu, X.; Zeng, N.; Liu, Y.; Alsaadi, F.E. A survey of deep neural network architectures and their applications. Neurocomputing 2017, 234, 11-26. [CrossRef] 
44. Guo, Y.; Liu, Y.; Oerlemans, A.; Lao, S.; Wu, S.; Lew, M.S. Deep learning for visual understanding: A review. Neurocomputing 2016, 187, 27-48. [CrossRef]

45. Simonyan, K.; Zisserman, A. Very Deep Convolutional Networks for Large-Scale Image Recognition. arXiv, 2014; arXiv:1409.1556.

46. Szegedy, C.; Liu, W.; Jia, Y.; Sermanet, P.; Reed, S.; Anguelov, D.; Erhan, D.; Vanhoucke, V.; Rabinovich, A. Going deeper with convolutions. In Proceedings of the IEEE Conference on Computer Vision and Pattern Recognition (CVPR), Boston, MA, USA, 7-12 June 2015; pp. 1-9.

47. Chollet, F. Xception: Deep Learning with Depthwise Separable Convolutions, CoRR abs/1610.02357. 2016. Available online: http:/ / arxiv.org/abs/1610.02357 (accessed on 16 August 2018).

48. He, K.; Zhang, X.; Ren, S.; Sun, J. Deep residual learning for image recognition. In Proceedings of the IEEE Conference on Computer Vision and Pattern Recognition (CVPR), Las Vegas, NV, USA, 27-30 June 2016; pp. 770-778.

49. Deng, J.; Dong, W.; Socher, R.; Li, L.-J.; Li, K.; Fei-Fei, L. Imagenet: A large-scale hierarchical image database. In Proceedings of the IEEE Conference on Computer Vision and Pattern Recognition (CVPR), Miami, FL, USA, 20-25 June 2009; pp. 248-255.

50. Eckersley, P.; Nasser, Y. AI Progress Measurement. Available online: https://www.eff.org/ai/metrics (accessed on 13 August 2018).

51. Deng, L.; Yu, D. Deep Learning: Methods and Applications. SIG 2014, 7, 197-387. [CrossRef]

52. Deng, L. A tutorial survey of architectures, algorithms, and applications for deep learning. APSIPA Trans. Signal Inf. Process. 2014, 3. [CrossRef]

53. Schmidhuber, J. Deep learning in neural networks: An overview. Neural Netw. 2015, 61, 85-117. [CrossRef] [PubMed]

54. Lazzara, M.A.; Keller, L.M.; Stearns, C.R.; Thom, J.E.; Weidner, G.A. Antarctic satellite meteorology: Applications for weather forecasting. Mon. Weather Rev. 2003, 131, 371-383. [CrossRef]

55. Kohrs, R.A.; Lazzara, M.A.; Robaidek, J.O.; Santek, D.A.; Knuth, S.L. Global satellite composites-20 years of evolution. Atmos. Res. 2014, 135-136, 8-34. [CrossRef]

56. Simard, P.Y.; Steinkraus, D.; Platt, J.C. Best practices for convolutional neural networks applied to visual document analysis. In Proceedings of the Seventh International Conference on Document Analysis and Recognition, Washington, DC, USA, 3-6 August 2003; IEEE: Edinburgh, Scotland, UK, 2003; p. 958.

57. Pratt, L.Y.; Mostow, J.; Kamm, C.A.; Kamm, A.A. Direct Transfer of Learned Information Among Neural Networks. In Advances in Neural Information Processing Systems; MIT Press: Cambridge, MA, USA, 1992; Volume 5, pp. 204-211.

58. Caruana, R. Learning Many Related Tasks at the Same Time with Backpropagation. In Advances in Neural Information Processing Systems; MIT Press: Cambridge, MA, USA, 1995; Volume 7.

59. Collobert, R.; Weston, J. A unified architecture for natural language processing: Deep neural networks with multitask learning. In Proceedings of the 25th International Conference on Machine Learning, Helsinki, Finland, 5-9 July 2008; ACM: New York, NY, USA, 2008; pp. 160-167.

60. Pan, S.J.; Yang, Q. A Survey on Transfer Learning. IEEE Trans. Knowl. Data Eng. 2010, 22, $1345-1359$. [CrossRef]

61. Mesnil, G.; Dauphin, Y.; Glorot, X.; Rifai, S.; Bengio, Y.; Goodfellow, I.; Lavoie, E.; Muller, X.; Desjardins, G.; Warde-Farley, D. Unsupervised and transfer learning challenge: A deep learning approach. Proc. Int. Conf. Unsupervised Transf. Learn. Workshop 2011, 27, 97-111.

62. Oquab, M.; Bottou, L.; Laptev, I.; Sivic, J. Learning and Transferring Mid-Level Image Representations using Convolutional Neural Networks. In Proceedings of the IEEE Conference on Computer Vision and Pattern Recognition, Columbus, OH, USA, 23-28 June 2014; pp. 1717-1724.

63. Maclin, R.; Shavlik, J.W. Combining the predictions of multiple classifiers: Using competitive learning to initialize neural networks. In Proceedings of the 1995 International Joint Conference on AI, Montreal, QC, Canada, 20-25 August 1995; Citeseer: Montreal, QC, Canada, 1995; pp. 524-531.

64. Srivastava, N.; Hinton, G.; Krizhevsky, A.; Sutskever, I.; Salakhutdinov, R. Dropout: A simple way to prevent neural networks from overfitting. J. Mach. Learn. Res. 2014, 15, 1929-1958.

65. Agakov, F.V.; Barber, D. An Auxiliary Variational Method. In Neural Information Processing; Lecture Notes in Computer Science; Springer: Berlin/Heidelberg, Germany, 2004; pp. 561-566. 
66. Rumelhart, D.E.; Hinton, G.E.; Williams, R.J. Learning representations by back-propagating errors. Nature 1986, 323, 533-536. [CrossRef]

67. Sorokin, A.A.; Makogonov, S.V.; Korolev, S.P. The Information Infrastructure for Collective Scientific Work in the Far East of Russia. Sci. Tech. Inf. Proc. 2017, 44, 302-304. [CrossRef]

68. Carleton, A.M.; Carpenter, D.A. Satellite climatology of 'polar lows' and broadscale climatic associations for the Southern Hemisphere. Int. J. Climatol. 1990, 10, 219-246. [CrossRef]

69. Carleton, A.M.; Fitch, M. Synoptic aspects of Antarctic mesocyclones. J. Geophys. Res. Atmos. 1993, 98, 12997-13018. [CrossRef]

70. Ronneberger, O.; Fischer, P.; Brox, T. U-Net: Convolutional Networks for Biomedical Image Segmentation. In Medical Image Computing and Computer-Assisted Intervention-MICCAI 2015; Lecture Notes in Computer Science; Springer: Cham, Switzerland, 2015; pp. 234-241.

71. Badrinarayanan, V.; Kendall, A.; Cipolla, R. SegNet: A Deep Convolutional Encoder-Decoder Architecture for Image Segmentation. arXiv, 2015; arXiv:1511.00561. [CrossRef] [PubMed]

72. Parkhi, O.M.; Vedaldi, A.; Zisserman, A. Deep Face Recognition. In Proceedings of the British Machine Vision Conference (BMVC); BMVA Press: Durham, UK, 2015; pp. 41.1-41.12.

73. Schroff, F.; Kalenichenko, D.; Philbin, J. FaceNet: A Unified Embedding for Face Recognition and Clustering. In Proceedings of the IEEE Conference on Computer Vision and Pattern Recognition (CVPR), Boston, MA, USA, 7-12 June 2015; pp. 815-823.

74. Minsky, M.; Papert, S.A. Perceptrons; MIT Press: Cambridge, MA, USA, 1969; ISBN 978-0-262-13043-1.

75. Breiman, L. Bagging predictors. Mach. Learn. 1996, 24, 123-140. [CrossRef]

76. Lincoln, W.P.; Skrzypek, J. Synergy of clustering multiple back propagation networks. In Advances in Neural Information Processing Systems; MIT Press: Cambridge, MA, USA, 1990; pp. 650-657.

(C) 2018 by the authors. Licensee MDPI, Basel, Switzerland. This article is an open access article distributed under the terms and conditions of the Creative Commons Attribution (CC BY) license (http:/ / creativecommons.org/licenses/by/4.0/). 